\title{
BMJ Open Efficacy of psychosocial interventions for psychological and pregnancy outcomes in infertile women and men: a systematic review and meta-analysis
}

\author{
Yoon Frederiksen, ${ }^{1}$ Ingeborg Farver-Vestergaard, ${ }^{1}$ Ninna Grønhøj Skovgård, ${ }^{1}$ \\ Hans Jakob Ingerslev, ${ }^{2}$ Robert Zachariae ${ }^{1,3}$
}

To cite: Frederiksen $\mathrm{Y}$, Farver-Vestergaard I, Skovgård NG, et al. Efficacy of psychosocial interventions for psychological and pregnancy outcomes in infertile women and men: a systematic review and meta-analysis. BMJ Open 2015;5:e006592.

doi:10.1136/bmjopen-2014006592

- Prepublication history and additional material is available. To view please visit the journal (http://dx.doi.org/ 10.1136/bmjopen-2014006592).

Received 11 September 2014 Revised 28 November 2014 Accepted 15 December 2014

CrossMark

For numbered affiliations see end of article.

Correspondence to Dr Yoon Frederiksen; yoonf@psy.au.dk

\section{ABSTRACT}

Objective: To evaluate the evidence on the efficacy of psychosocial interventions for improving pregnancy rates and reducing distress for couples in treatment with assisted reproductive technology (ART).

Design: Systematic review and meta-analysis.

Data sources: PsycINFO, PubMed, EMBASE, CINAHL, Web of Science and The Cochrane Library between 1978 and April 2014.

Study selection: Studies were considered eligible if they evaluated the effect of any psychosocial intervention on clinical pregnancy and/or distress in infertile participants, used a quantitative approach and were published in English.

Data extraction: Study characteristics and results were extracted and the methodological quality was assessed. Effect sizes (ES; Hedges g) were pooled using a random effects model. Heterogeneity was assessed using the $Q$ statistic and $\mathrm{I}^{2}$, and publication bias was evaluated using Egger's method. Possible moderators and mediators were explored with metaanalyses of variances (ANOVAs) and meta-regression.

Results: We identified 39 eligible studies (total $\mathrm{N}=2746$ men and women) assessing the effects of psychological treatment on pregnancy rates and/or adverse psychological outcomes, including depressive symptoms, anxiety, infertility stress and marital function. Statistically significant and robust overall effects of psychosocial intervention were found for both clinical pregnancy (risk ratio $=2.01 ; \mathrm{Cl} 1.48$ to 2.73; $p<0.001$ ) and combined psychological outcomes (Hedges $\mathrm{g}=0.59 ; \mathrm{Cl} 0.38$ to $0.80 ; \mathrm{p}=0.001$ ). The pooled ES for psychological outcomes were generally larger for women ( $\mathrm{g}: 0.51$ to 0.73 ) than men (0.13 to 0.34 ), but the difference only reached statistical significance for depressive symptoms $(p=0.004)$. Meta-regression indicated that larger reductions in anxiety were associated with greater improvement in pregnancy rates (Slope $0.19 ; p=0.004$ ). No clear-cut differences were found between effects of cognitive-behavioural therapy (CBT; $g=0.84)$, mind-body interventions $(0.61)$ and other intervention types $(0.50)$.

Conclusions: The present meta-analysis suggests that psychosocial interventions for couples in treatment for infertility, in particular CBT, could be efficacious, both

\author{
Strengths and limitations of this study
}

A major strength of this study is the extensive search of various databases from 1978 to April 2014, as well as a comprehensive methodological assessment.

- Further analyses were performed to account for publication bias, yielding conservative effect sizes and thus strengthening the robustness of the estimates.

- Heterogeneity and indications of publication bias were observed for several of the outcomes.

- A substantial variation of the methodological quality and missing information on fertility and assisted reproductive technology (ART) treatment may limit the interpretability of the outcomes.

in reducing psychological distress and in improving clinical pregnancy rates.

\section{INTRODUCTION}

Fecundity has become a growing problem for many couples trying to conceive a child, and although not all couples choose to seek medical assistance, more than $10 \%$ of the childbearing population has resorted to assisted reproductive technology (ART) to conceive. ${ }^{1-5}$ Being involuntarily childless and going through various ART procedures imposes considerable stress on the couple, and childlessness is often perceived as a life crisis where the emotional strain equals that found for traumatic events. ${ }^{2}$ 6-10 Although infertile couples may be considered mentally healthy in general, ${ }^{11}$ several studies indicate that coping with infertility is associated with periodically heightened levels of psychological symptoms of distress, depression and anxiety. ${ }^{12}{ }^{13}$ Feelings of loss, grief, anger and sadness are not uncommon, and women 
often report bodily disparagement, lack of femininity, shame and self-blame. ${ }^{2} 14$ There is some evidence to suggest that dysregulation in the uterus microenvironment may influence the ability to conceive, for example, oxidative stress and inflammation, ${ }^{15}{ }^{16}$ which may be promoted by psychological distress. ${ }^{17} 18$ Such findings have led several studies to investigate possible links between mental state and pregnancy outcome. ${ }^{10}$ 19-24 Although the results have been mixed, reviews of the literature have generally reached the conclusion that psychosocial factors such as depressive symptoms, anxiety, distress and certain coping strategies are linked to reduced chances of pregnancy. ${ }^{12} 2526$ Two recently published meta-analyses, however, report conflicting results. ${ }^{27} 28$ Whereas one meta-analysis supported the conclusion that emotional distress may be critical to the success of fertility treatment outcome, ${ }^{27}$ the other did not find sufficient support for this hypothesis. ${ }^{28}$ The different conclusions could be due to between-study methodological differences, for example, in the chosen measures of distress and definitions of pregnancy (eg, serum positive test, clinical pregnancy or live birth).

Nonetheless, the evidence indicating a considerable psychosocial burden associated with infertility and its treatment has inspired several researchers to explore the effect of various psychosocial interventions in reducing distress, improving quality of life, and thereby possibly optimising the chances of pregnancy. So far, three meta-analyses have reviewed effects of psychological interventions on mental health and pregnancy outcome. Again, the results have been mixed. The first meta-analysis, published in 2003, concluded that psychological intervention appeared to have a beneficial effect on negative emotions, ${ }^{29}$ particularly anxiety. An effect of counselling was also found for infertility-related distress, whereas no clear effect was seen on pregnancy rates. Although the original systematic review identified 25 independent studies, the final meta-analysis only included 8-10 studies selected on the basis of their methodological quality. The second meta-analysis published in 2005 focused on differences in effects related to intervention format, for example, individual/couple versus group setting. ${ }^{30}$ Overall, the results suggested that both individual/couple and group interventions were effective in reducing emotional distress as well as increasing the conception rate. In contrast to the two first meta-analyses, which had investigated both controlled and uncontrolled studies, the third meta-analysis from 2009, which only included controlled studies, ${ }^{31}$ found no evidence for an effect of psychological interventions on emotional distress. An effect, however, was found for pregnancy rates, but only for infertile couples not in ART.

Taken together, while showing promising results, the findings of existing quantitative systematic reviews, the most recent published in 2009, are mixed. The literature within this field is expanding, and studies of new psychosocial intervention approaches building on existing knowledge and targeting specific problems of infertile patients, for example, mind/body interventions (MBIs), internet-based treatments and online psychoeducation programmes, have since been published. Furthermore, the more recently published studies have generally used randomised controlled trial (RCT) designs, a notable strength reducing the risk of bias and making the studies more easily comparable. ${ }^{32}$ An updated review and meta-analysis is needed to determine to what degree psychosocial interventions may reduce infertility-related distress related to improvement of pregnancy chances during fertility treatment.

\section{METHODS}

The present study was conducted in accordance with the preferred reporting items for systematic reviews and meta-analyses (PRISMA) recommendations. ${ }^{33}{ }^{34}$ An a priori designed study protocol guided the literature search, study selection and data synthesis.

\section{Search strategy and criteria}

A comprehensive and systematic search of the literature published between 1978 (first baby born after in vitro fertilisation (IVF)) and April 2014 was conducted, using a sensitive search strategy recommended for reviews by Higgins and Green. ${ }^{35}$ When conducting the searches, we combined keywords representing the two primary concepts, infertility and psychosocial treatment: (1) "infertil*", "childlessness", "IVF", "ICSI", "fertility treatment/problems" "assisted reproduction" and (2) "psychological/psychosocial intervention", "social support", "couples therapy", "psycho-education", "internet-based intervention" and "behavioral therapy" (for a full search history, see online supplementary appendix 1). We identified relevant records by electronic searches in general medical and psychological databases: PubMed, PsycINFO, The Cochrane Library, EMBASE, CINAHL and Web of Science. Furthermore, we cross-examined reference lists of the retrieved papers and reviews for additional relevant studies. We did not pursue the grey literature or trial registries, and limited our search to include only peer-reviewed articles published in English.

\section{Study selection}

Studies were considered eligible if they (1) reported data on infertile participants (2) presented data on a psychosocial intervention or a supportive programme (3) included baseline and postintervention measures of stress, distress or pregnancy outcome and (4) used a quantitative research approach. In general terms, infertility refers to not being able to conceive for more than 1 year without contraception (WHO, 2002). Despite this standard definition, a recent review has found considerable between-study variation in definitions. ${ }^{36}$ Furthermore, infertility can be graded in relation to clinical diagnosis and duration. The present meta-analysis reviews studies using several different definitions of the term 'infertile', and includes all studies of patients 
diagnosed with different types of infertility and in different types and stages of ART treatments, for example, intrauterine insemination (IUI), IVF and intracytoplasmic sperm injection (ICSI). 'Psychosocial interventions or supportive programs' were defined as all interventions with a psychosocial aim that did not include the prescription of medication or had a primary physical focus, for example, acupuncture or massage therapy. However, studies using 'psychophysiological' approaches, for example, relaxation, guided imagery or meditation exercises as part of a psychosocial programme, were included. The interventions could be delivered in an individual-based, group-based, couples-based or internetbased format. We included controlled and uncontrolled trial (UCT) studies, but chose to exclude expert opinion, magazines, commentaries, case reports, editorials, newspaper articles, newsletters and book chapters. Neither did we include abstracts-only, doctoral theses or conference presentations. Our primary outcome was pregnancy rate, defined as clinical pregnancy. This clinical definition implies a visualisation of at least one gestational sac and fetal heartbeat in approximately the fifth week after fertilisation. Secondary outcome measures were psychological ratings of depressive symptoms, anxiety, generalised stress, specific infertility stress and interpersonal functioning assessed through self-reported questionnaires.

\section{Data extraction and quality assessment}

All full-text articles were read by two independent review authors (IF-V, NGS) and the data were extracted according to predefined criteria. Disagreements were discussed with a third author (YF) and resolved by consensus. If information on any outcome was missing or if clarifications were needed, authors were contacted for further information. Each study was assessed for methodological quality using the Jadad criteria, ${ }^{37}$ a commonly used tool to evaluate methodological quality, for example, use and adequate description of randomisation and blinding procedures, and description of dropout rates (score range $0-5$ ). In addition to the $0-5$ points possible on the original Jadad scale, 1 additional point was given for each of the following: (1) was a control group included, in order to acknowledge whether the intervention group was compared with another group, although randomisation was not used; (2) were both predata and postdata presented, as including preintervention and postintervention data will provide more accurate results; (3) was any form of blinding or masking of conditions to patients or (4) blinding of researchers attempted, acknowledging if the study had attempted to mask the active condition; (5) was a standardised and reliable outcome measure used, a criterion increasing the validity and comparability of the outcomes and (6) were pre-post correlations provided, which could provide better estimates of the effect size (ES). The modified scale yielded a total quality score ranging from 0 to 11 . With respect to the modified quality score, the mean score difference between rater 1 and 2 (means (SD) $5.2(1.8)$ and $5.6(2.0))$ did not reach statistical significance $(\mathrm{t}(77)=1.1 ; \mathrm{p}=0.28)$, and the inter-rater score correlation was $\mathrm{r}=0.83(\mathrm{p}<0.001)$. The $\kappa$ Statistic was not used, as this assumes the nominal data and no natural ordering of ratings. Quality ratings were not used as weights when calculating aggregated ES as this is generally discouraged due to the risk of introducing additional bias. ${ }^{38}$ Instead, associations between ES and study quality indicators were explored with meta-analyses of variances (ANOVAs) and meta-regression (modified quality score). In cases where we were unable to retrieve articles from the authorised databases, authors were contacted between 1 and 3 times in order to amend the data collected.

\section{Calculating ES}

The ES used were the risk ratio (RR) for pregnancy and Hedges $\mathrm{g}$ for psychological outcomes. Hedges $\mathrm{g}$ is a variation of Cohen's $d$ which enables correction of potential bias due to small sample sizes. ${ }^{39} 40$ A positive Hedges $g$ indicates a result in the expected direction, for example, a reduction in distress in the intervention group compared with controls. An RR $>1.0$ indicates a greater proportion of pregnancies in the intervention group. RRs were based on pregnancy rates and total $\mathrm{N}$ in the intervention and control groups. When possible, Hedges $g$ was calculated on the basis of reported means and SDs at preintervention and postintervention or means and SDs of change scores. This was possible for 50 of $61 \mathrm{ES}$. When required and available, the reported pre-post correlations were used in the calculation. This was the case for five ES. When unavailable, the pre-post correlation was set to 0.50 . When SDs were unavailable, two approaches were used. For STAI (State-Trait Anxiety Inventory) state anxiety scores, the average pre-SDs and post-SDs (10.9 and 10.8, respectively) for the studies which reported the SD were used, as the SDs appeared to be highly comparable across the remaining studies. For other measures, ES were estimated either on the basis of sample size and either $p$ value or $\eta^{2}$. In one study reporting only medians, ${ }^{41}$ the means and SDs were estimated following a previously suggested approach. ${ }^{42}$

\section{Heterogeneity}

Heterogeneity was assessed using $Q$ and $\mathrm{I}^{2}$ statistics. Heterogeneity tests are aimed at determining whether results reflect genuine between-study differences (heterogeneity), or whether the variation is due to chance (homogeneity). ${ }^{43}$ In accordance with recommendations, a $\mathrm{p}$ value $\leq 0.10$ was used to determine significant heterogeneity due to the general low statistical power of heterogeneity tests. ${ }^{44}$ The $\mathrm{I}^{2}$ quantity provides a measure of the degree of inconsistency by estimating the amount of variance in a pooled ES that can be accounted for by heterogeneity in the sample of studies. ${ }^{45} \mathrm{I}^{2}$ values of $0 \%$, $25 \%, 50 \%$ and $75 \%$ indicate no, low, moderate and high heterogeneity, respectively. 


\section{Analytical strategy}

All ES were weighted with the inverse variance and combined with a random effects model. First, the overall ES of the effect of psychosocial interventions on pregnancy rates was calculated. Then the overall ES for the combined psychological outcomes was calculated together with the overall ES for the individual outcome measures of depression, state anxiety, infertility-related distress and marital function. This was performed for the combined sample (women+men). If the results indicated study heterogeneity, and if the number of studies in each category was sufficient $(\mathrm{K} \geq 3)$, possible between-study differences in ES were explored by comparing the ES of studies according to the following study characteristics: gender, study design, intervention type and intervention format (mixed effect meta-ANOVAs), methodological quality (modified quality score), mean age of the sample, intervention duration and number of sessions (mixed effect meta-regression).

Prior to the search, statistical power analyses were conducted as previously recommended. ${ }^{46}$ On the basis of the findings of the earlier meta-analysis, ${ }^{31}$ we expected to find an RR of 1.4 for pregnancy rates and an average sample size of $\mathrm{N}=76$. We expected to be able to detect a similar small ES (Hedges $\mathrm{g}=0.28$ or $\mathrm{RR}=1.4$ ) with an $\alpha$ of $5 \%$ and a statistical power of $80 \%$, with a total of only nine studies, using a random effects model. On the basis of these results, we considered it worthwhile to conduct the meta-analysis. The calculations were conducted using Comprehensive Meta-Analysis, V.2 (http://www. meta-analysis.com), IBM SPSS V.20 and various formulas in Microsoft Excel.

\section{Publication bias}

The possibility of publication bias, a widespread problem when conducting meta-analyses, was evaluated with funnel plots, ${ }^{47}$ Egger's method and by calculating fail-safe numbers. ${ }^{48} 49$ A funnel plot is a graphic illustration of study ES in relation to study size or precision. Egger's test provides a statistic for the skewness of results. ${ }^{50}$ Calculation of fail-safe numbers is aimed at achieving an indication of the number of unpublished studies with null findings that would reduce the result to statistical non-significance $(p>0.05)$. It has been suggested that a reasonable level is achieved if the fail-safe number exceeds $5 \mathrm{~K}+10 \quad(\mathrm{~K}=\mathrm{N}$ studies in the meta-analysis).$^{51}$ If the results were suggestive of publication bias, an adjusted ES was calculated using Duval and Tweedie's $^{52}$ trim and fill method, which imputes ES of missing studies and recalculates the ES accordingly.

\section{RESULTS}

\section{Study selection}

In a first screening, duplicates were identified, and titles and abstracts reviewed. A total of 157 studies were found potentially relevant and reviewed independently by two raters. Four articles could not be retrieved due to the 'no access policy from the university, and the authors did not respond to our enquiries. ${ }^{53-56}$ Initially, the raters were uncertain or disagreed on $13(8.3 \%)$ articles (interrater agreement $0.78 ; \mathrm{p}<0.001$ ( $\kappa$ statistic) ) indicating 'substantial agreement'. ${ }^{57}$ After negotiation, 5 of these were included, resulting in 41 potentially eligible articles. One additional study was excluded due to the combination of psychological intervention with a psychoactive drug, and one study had insufficient statistical data and the authors did not respond to our enquiry. We thus included a total of 39 studies in the present review. On three occasions, the authors provided unpublished additional data. ${ }^{58-60}$ Figure 1 shows a flow chart of the study selection process.

\section{Study characteristics}

The study characteristics are summarised in table 1 . Based on the outcome, 29 of the studies were aimed at reducing negative emotional distress, ${ }^{41}{ }^{58-85}$ with the targeted outcomes being infertility-related distress $(\mathrm{k}=10)$, depression $(\mathrm{k}=21)$, anxiety $(\mathrm{k}=25)$ and marital function $(k=5)$. Five studies focused solely on the outcome of pregnancy ${ }^{86-90}$ and five had included distress as well as pregnancy as the outcome. ${ }^{78}{ }^{91-94}$ Twenty-one studies were RCTs, ${ }^{58} \quad 61 \quad 65-72 \quad 74 \quad 75 \quad 83 \quad 8589-95$ and 10 were non-RCTs (NRCTs), ${ }^{41} 59 \quad 6076 \quad 79 \quad 8086-88 \quad 96$ with most control groups receiving standardised care or being waiting list controls. Only three studies had included an active/attention control condition, for example, non-emotional writing or receiving an information booklet. $^{707174}$ One study offered gift certificates to the control group participants if they responded to the follow-up questionnaires. ${ }^{89}$ Relatively few studies were UCTs $(\mathrm{k}=8) .{ }^{62-64} 7377818284$ The reporting of the participants' medical treatment status was inconsistent. Five studies did not provide information on treatment status (whether or not in current ART treatment), 3 reported that some, but not how many, of the participants were in treatment, and 31 reported that their participants were currently in ART treatment, although not what kind of treatment, for example, IUI, IVF/ICSI or treatment cycle. The cause of infertility was also inconsistently reported, and some participants may still have been under evaluation during the study period. Twenty-five studies had included only women, while the remaining 14 had included both women and men. The included studies had reported data for a total of 3401 participants (3064 women and 347 men). The mean age and mean duration of infertility for intervention group participants were (32.7 years, 'SD' 2.2) and (4.6 years, 'SD' 2.1), and for control group participants (32.6 years, 'SD' 1.7) and (5.1 years, 'SD' 3.0), respectively. The specific intervention strategies mostly employed were cognitive-behavioural therapy (CBT; $\mathrm{k}=8)$ and MBI $(\mathrm{k}=12)$. The remaining studies had used a variety of interventions, including stress management, hypnosis, art therapy, expressive writing intervention, crisis intervention and various types of counselling. Some studies had included 


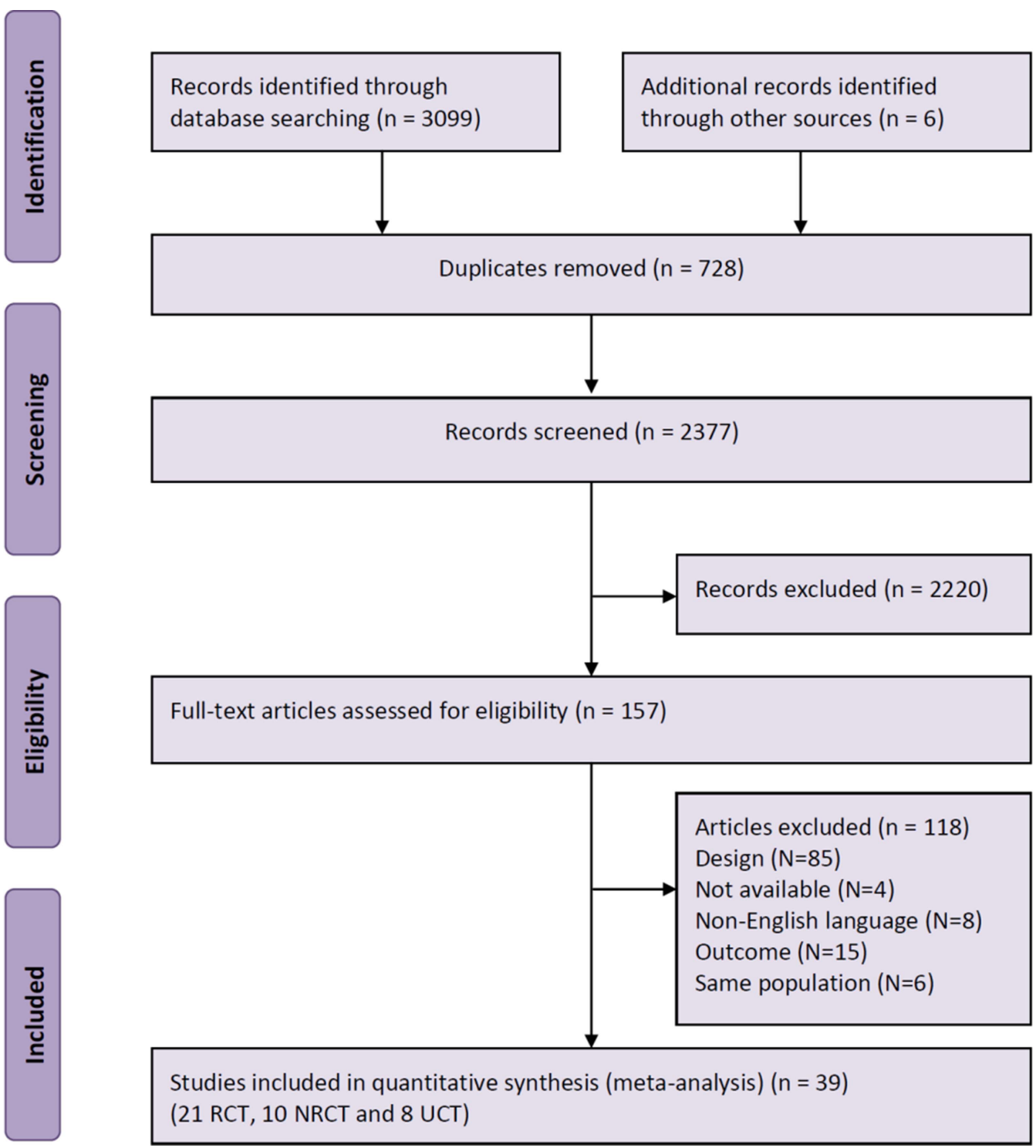

Figure 1 PRISMA flow chart of selection of studies (NRCT, non-randomised controlled trial; PRISMA, Preferred Reporting Items for Systematic Reviews and Meta-Analyses; RCT, randomised controlled trial; UCT, uncontrolled trial).

more than one approach, for example, cognitive-behavioural approaches supplemented with mind-body techniques such as relaxation. To be categorised as MBI, a study had to use such strategies as the general approach over the course of intervention. Thus, if studies had mainly used CBT strategies and only incorporated other approaches, for example, relaxation exercises, in one or two sessions, they were categorised as CBT interventions. The number of sessions ranged from 1 to 24 , lasting approximately from $20 \mathrm{~min}$ to $3 \mathrm{~h}$, and the duration of psychosocial intervention ranged from 1 week to 28 months.

\section{Attrition}

A total of 15 studies reported the number of participants at baseline and then again at follow-up, and as seen in table 1, the number of dropouts varied across studies. Although the dropout rates in the intervention groups were somewhat higher (mean $30.5 \%$ (SD 20.2)) than in controls $(24.9 \%(24.8))$, the difference did not reach statistical significance $(\mathrm{t}(28) 0.68, \mathrm{p}=0.50)$. Furthermore, only four studies explicitly stated that the analysis was based on an intention-to-treat (ITT) approach. ${ }^{70} 728392$ Two additional studies used methods comparable to ITT, for example, carrying last (baseline) observations forward or use of multilevel linear modelling. ${ }^{6997}$ Four studies stated that there were no differences between completers and dropouts without specifying this further, $^{41} 648185$ and the remaining studies failed to report whether there were dropouts or how such missing data were dealt with. The possible association between ES and uneven dropouts in the intervention and control groups was analysed for the 15 studies that reported dropouts by regressing the difference in dropout rates on the overall ES across all outcomes. The result indicated that larger dropouts in the intervention group 
Table 1 Characteristics of included studies

\begin{tabular}{|c|c|c|c|c|c|c|c|c|c|c|c|}
\hline Author & Country & $\begin{array}{l}\text { Participants (N) } \\
\text { I: intervention } \\
\text { C: control } \\
\text { assigned } \\
\text { (final analysis) } \\
\text { (men \%) }\end{array}$ & $\begin{array}{l}\text { Study } \\
\text { design }\end{array}$ & Intervention type ${ }^{\star}$ & $\begin{array}{l}\text { Intervention } \\
\text { category† }\end{array}$ & $\begin{array}{l}\text { Intervention } \\
\text { format }\end{array}$ & $\begin{array}{l}\text { Number of } \\
\text { sessions }\end{array}$ & $\begin{array}{l}\text { Intervention } \\
\text { duration } \\
\text { (weeks) }\end{array}$ & $\begin{array}{l}\text { Outcome: } \\
\text { psychologicalł } \\
\text { IS: infertility stress } \\
\text { A: anxiety } \\
\text { D: depression } \\
\text { MF: marital function }\end{array}$ & $\begin{array}{l}\text { Outcome: } \\
\text { pregnancy§ } \\
(+/-)\end{array}$ & $\begin{array}{l}\text { Quality scoreף } \\
\text { J: Jadad 0-5 } \\
\text { MJ: modified } \\
\text { Jadad 0-12 } \\
\text { J (MJ) }\end{array}$ \\
\hline O'Moore et $a l^{79}$ & Ireland, UK & $\begin{array}{l}\text { I: } 30(22)(50 \%) \\
\text { C: } 20(20)(50 \%)\end{array}$ & NRCT & Autogenic training & $\mathrm{MBI}$ & Group & 8 & 8 & $\begin{array}{l}\text { D: BDI } \\
\text { A: STAI }\end{array}$ & - & $1(4)$ \\
\hline Lukse ${ }^{62}$ & USA & I: $29(29(14))$ & UCT & Counselling & Other & Group & 6 & 6 & D: DES & - & $0(3)$ \\
\hline $\begin{array}{l}\text { Sarrel and } \\
\text { DeCherney }\end{array}$ & USA & $\begin{array}{l}\text { I: } 20(10) \\
\text { C: } 20(9)\end{array}$ & NRCT & $\begin{array}{l}\text { Psychotherapeutic } \\
\text { interview }\end{array}$ & Other & Couples & 1 & 1 & & + & $0(1)$ \\
\hline Domar et $a^{\rho^{3}}$ & USA & I: 54 (54) & UCT & Mind/body programme & $\mathrm{MBI}$ & Group & 10 & 10 & A: STAI & - & 0 (3) \\
\hline Domar et $a{ }^{4}$ & USA & I: $52(41)$ & UCT & $\begin{array}{l}\text { Behavioural medicine } \\
\text { programme for infertility }\end{array}$ & $\mathrm{MBI}$ & Group & 10 & 10 & A: STAI & - & $1(3)$ \\
\hline Galletly et $a^{\beta^{2}}$ & Australia & I: 37 (37) & UCT & Treatment programme & Other & Group & 24 & 24 & $\begin{array}{l}\text { D: HADS } \\
\text { A: HADS }\end{array}$ & - & $1(3)$ \\
\hline McQueeney et $a^{\beta 0}$ & USA & $\begin{array}{l}\text { I: } 20(20) \\
\text { C: } 9 \text { (9) }\end{array}$ & NRCT & $\begin{array}{l}\text { Emotion-focused and } \\
\text { problem-focused } \\
\text { therapies }\end{array}$ & Other & Group & 6 & 6 & $\begin{array}{l}\text { IS: ISD } \\
\text { D: BDI }\end{array}$ & - & $3(7)$ \\
\hline $\begin{array}{l}\text { Tuschen-Caffier } \\
\text { et } a f^{41}\end{array}$ & Germany & $\begin{array}{l}\text { I: } 34(22) \\
\text { C: } 24(24)\end{array}$ & NRCT & СBT & CBT & Couples & $10-12$ & 32 & $\begin{array}{l}\text { IS: one item } \\
\text { MF: one item }\end{array}$ & - & $1(4)$ \\
\hline Domar et al ${ }^{7895}$ & USA & $\begin{array}{l}\text { I: } 56(20) \\
\text { C: } 63(14)\end{array}$ & $\mathrm{RCT}$ & $\begin{array}{l}\text { Psychological } \\
\text { intervention }\end{array}$ & $\mathrm{MBI}$ & Group & 10 & 10 & $\begin{array}{l}\text { D: BDI } \\
\text { A: STAI }\end{array}$ & + & $4(10)$ \\
\hline Terzioglu ${ }^{91}$ & Turkey & $\begin{array}{l}\text { I: } 60(60)(50 \%) \\
\text { C: } 60(60)(50 \%)\end{array}$ & $\mathrm{RCT}$ & Counselling & Other & Individual & 5 & 5 & $\begin{array}{l}\text { D: BDI } \\
\text { A: STAI }\end{array}$ & + & $2(5)$ \\
\hline Hosaka et a $\beta^{77}$ & Japan & $\begin{array}{l}\text { I: } 37(37) \\
\text { C: } 37(37)\end{array}$ & NRCT & Structured intervention & $\mathrm{MBI}$ & Group & 5 & 5 & & + & $3(6)$ \\
\hline $\begin{array}{l}\text { McNaughton-Cassill } \\
\text { et } a^{\beta^{6}}\end{array}$ & USA & $\begin{array}{l}\text { I: } 43(43)(39.5 \%) \\
\text { C: } 37(37)(48.6 \%)\end{array}$ & $\mathrm{NRCT}$ & Couples' support & CBT & Couples & 6 & 3 & $\begin{array}{l}\text { D: BDI } \\
\text { A: BAI }\end{array}$ & - & $2(5)$ \\
\hline Emery et $a^{{ }^{5}}$ & Switzerland & $\begin{array}{l}\text { I: } 158(110) \\
(34.8 \%) \\
\text { C: } 152(131) \\
(42.8 \%)\end{array}$ & $\mathrm{RCT}$ & Pre-IVF counselling & Other & Couples & 1 & 1 & $\begin{array}{l}\text { D: BDI } \\
\text { A: STAI }\end{array}$ & - & $3(6)$ \\
\hline $\operatorname{Lee}^{66}$ & Taiwan & $\begin{array}{l}\text { I: } 64(64) \\
\text { C: } 68(68)\end{array}$ & $\mathrm{RCT}$ & $\begin{array}{l}\text { Nursing crisis } \\
\text { intervention programme }\end{array}$ & MBI & Individual & 7 & 7 & $\begin{array}{l}\text { D: SDS } \\
\text { A: STAI }\end{array}$ & - & $1(4)$ \\
\hline De Klerk et $a^{{ }^{2}}$ & $\begin{array}{l}\text { The } \\
\text { Netherlands }\end{array}$ & $\begin{array}{l}\text { I: } 22(18) \\
\text { C: } 22(15)\end{array}$ & $\mathrm{RCT}$ & Counselling & Other & Group & 3 & $4-5$ & $\begin{array}{l}\text { D: HADS } \\
\text { A: HADS }\end{array}$ & - & $3(6)$ \\
\hline Schmidt et $a^{\rho^{9}}$ & Denmark & $\begin{array}{l}\text { I: } 13(13) \\
\text { C: } 435(435)\end{array}$ & NRCT & Stress management & Other & Group & 5 & 6 & IS: COMPI & - & $1(4)$ \\
\hline Chan et $a^{\rho 7}$ & $\begin{array}{l}\text { Hong Kong, } \\
\text { China }\end{array}$ & $\begin{array}{l}\text { I: } 101(69) \\
\text { C: } 126(115)\end{array}$ & $\mathrm{RCT}$ & $\begin{array}{l}\text { The Eastern body-mind } \\
\text { intervention }\end{array}$ & $\mathrm{MBI}$ & Group & 4 & 4 & A: STAI & - & $3(7)$ \\
\hline Levitas et $a^{\beta 8}$ & Israel & $\begin{array}{l}\text { I: } 89(89) \\
\text { C: } 96(96)\end{array}$ & NRCT & Hypnosis & MBI & Individual & 1 & 1 & & + & $0(1)$ \\
\hline Nilforooshan et a ${ }^{p^{8}}$ & Iran & $\begin{array}{l}\text { I: } 30(30)(50 \%) \\
\text { C: } 30(30)(50 \%)\end{array}$ & $\mathrm{RCT}$ & $\begin{array}{l}\text { Cognitive-behavioural } \\
\text { counselling }\end{array}$ & CBT & Group & 6 & 6 & $\begin{array}{l}\text { D: BDI } \\
\text { A: BAI }\end{array}$ & - & $2(6)$ \\
\hline Tuil et $a^{{ }^{8}}$ & $\begin{array}{l}\text { The } \\
\text { Netherlands }\end{array}$ & $\begin{array}{l}\text { I: } 108(102)(50 \%) \\
\text { C: } 96(78)(48.7 \%)\end{array}$ & $\mathrm{RCT}$ & $\begin{array}{l}\text { Internet-based health } \\
\text { record }\end{array}$ & Other & Individual & Infinite & 2 & $\begin{array}{l}\text { D: BDI } \\
\text { A: STAI }\end{array}$ & + & $3(6)$ \\
\hline Cousineau et $a{ }^{\beta 3}$ & USA & $\begin{array}{l}\text { I: } 96(49) \\
\text { C: } 92(49)\end{array}$ & $\mathrm{RCT}$ & $\begin{array}{l}\text { Psycho-educational } \\
\text { support }\end{array}$ & Other & Online & $1-2$ & 4 & $\begin{array}{l}\text { IS: FPI } \\
\text { MF: RDAS }\end{array}$ & - & $4(8)$ \\
\hline Faramarzi et $a^{61}$ & Iran & $\begin{array}{l}\text { I: } 42(29) \\
\text { C: } 40(30)\end{array}$ & $\mathrm{RCT}$ & СBT & CBT & Group & 10 & 10 & $\begin{array}{l}\text { D: BDI } \\
\text { A: Cattell }\end{array}$ & - & $3(6)$ \\
\hline $\begin{array}{l}\text { Lancastle and } \\
\text { Boivin }^{69}\end{array}$ & Wales, UK & $\begin{array}{l}\text { I: } 28(28) \\
\text { C: } 27(27)\end{array}$ & $\mathrm{RCT}$ & Brief coping intervention & Other & Individual & 14 & 2 & IS: CIQ & - & $4(8)$ \\
\hline
\end{tabular}

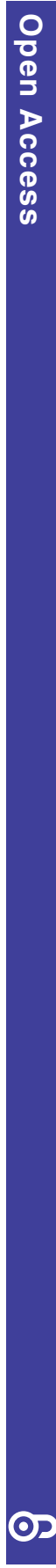

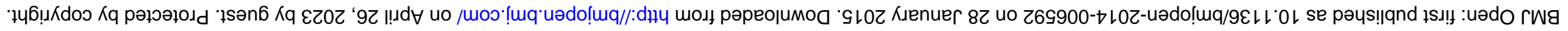




\begin{tabular}{|c|c|c|c|c|c|c|c|c|c|c|c|}
\hline Author & Country & $\begin{array}{l}\text { Participants (N) } \\
\text { I: intervention } \\
\text { C: control } \\
\text { assigned } \\
\text { (final analysis) } \\
\text { (men \%) }\end{array}$ & $\begin{array}{l}\text { Study } \\
\text { design }\end{array}$ & Intervention type ${ }^{\star}$ & $\begin{array}{l}\text { Intervention } \\
\text { category }\end{array}$ & $\begin{array}{l}\text { Intervention } \\
\text { format }\end{array}$ & $\begin{array}{l}\text { Number of } \\
\text { sessions }\end{array}$ & $\begin{array}{l}\text { Intervention } \\
\text { duration } \\
\text { (weeks) }\end{array}$ & $\begin{array}{l}\text { Outcome: } \\
\text { psychologicalł } \\
\text { IS: infertility stress } \\
\text { A: anxiety } \\
\text { D: depression } \\
\text { MF: marital function }\end{array}$ & $\begin{array}{l}\text { Outcome: } \\
\text { pregnancy§ } \\
(+/-)\end{array}$ & $\begin{array}{l}\text { Quality scoreף } \\
\text { J: Jadad 0-5 } \\
\text { MJ: modified } \\
\text { Jadad 0-12 } \\
\text { J (MJ) }\end{array}$ \\
\hline Noorbala et $a \beta^{\beta 4}$ & Iran & I: $288(288)(50 \%)$ & UCT & CBT & CBT & Group & & 24 & D: BDI & - & $3(8)$ \\
\hline Mori ${ }^{70}$ & Japan & $\begin{array}{l}\text { I: } 85(85) \\
\text { C: } 40(40)\end{array}$ & $\mathrm{RCT}$ & Stress management & Other & Individual & 3 & 12 & $\begin{array}{l}\text { D: HADS } \\
\text { A: HADS }\end{array}$ & - & $4(8)$ \\
\hline Panagopoulou et $a l^{71}$ & England, UK & $\begin{array}{l}\text { I: } 50(50) \\
\text { C: } 98(98)\end{array}$ & $\mathrm{RCT}$ & $\begin{array}{l}\text { Expressive writing } \\
\text { intervention }\end{array}$ & Other & Individual & 3 & 1 & $\begin{array}{l}\text { IS: ISS } \\
\text { A: STAI }\end{array}$ & + & $3(7)$ \\
\hline Haemmerli et $a l^{72}$ & Switzerland & $\begin{array}{l}\text { I: } 60(46) \\
\text { C: } 64(41)\end{array}$ & $\mathrm{RCT}$ & Coaching and support & Other & Online & 13 & 8 & $\begin{array}{l}\text { IS: IDS } \\
\text { D: CES-D } \\
\text { A: STAI }\end{array}$ & - & $3(6)$ \\
\hline Sexton et $a^{\beta 5}$ & USA & $\begin{array}{l}\text { I: } 21(15) \\
\text { C: } 22(16)\end{array}$ & $\mathrm{RCT}$ & $\begin{array}{l}\text { Web-based coping with } \\
\text { infertility }\end{array}$ & Other & Individual & & 2 & IS: FPI & - & $3(6)$ \\
\hline Domar et a $\beta^{9}$ & USA & $\begin{array}{l}\text { I: } 46(46) \\
\text { C: } 51(51)\end{array}$ & $\mathrm{RCT}$ & $\begin{array}{l}\text { Mind/body programme } \\
\text { for infertility }\end{array}$ & MBI & Group & 10 & 10 & & + & $4(6)$ \\
\hline $\begin{array}{l}\text { Hughes and de } \\
\text { Silva }^{73}\end{array}$ & Canada & I: $21(21)$ & UCT & Art therapy & Other & Group & $8(2 \mathrm{~h})$ & 8 & $\begin{array}{l}\text { D: BDI } \\
\text { A: BAI }\end{array}$ & - & $0(2)$ \\
\hline Chan et $a^{\beta^{4}}$ & $\begin{array}{l}\text { Hong Kong, } \\
\text { China }\end{array}$ & $\begin{array}{l}\text { I: } 141(141) \\
\text { C: } 110(110)\end{array}$ & $\mathrm{RCT}$ & $\begin{array}{l}\text { Integrative body-mind- } \\
\text { spirit intervention }\end{array}$ & $\mathrm{MBI}$ & Group & $4(3 \mathrm{~h})$ & 4 & $\begin{array}{l}\text { A: STAI } \\
\text { MF: C-KMS }\end{array}$ & + & $3(6)$ \\
\hline Gorayeb et $a^{\rho^{0}}$ & Brazil & $\begin{array}{l}\text { I: } 93(93) \\
\text { C: } 95(95)\end{array}$ & $\mathrm{RCT}$ & $\begin{array}{l}\text { Brief cognitive- } \\
\text { behavioural intervention }\end{array}$ & CBT & Group & $5(2 \mathrm{~h})$ & 5 & & + & $1(4)$ \\
\hline Koszycki et $a^{\beta 1}$ & Canada & I: 31 (23) & UCT & $\begin{array}{l}\text { Interpersonal and } \\
\text { supportive therapy }\end{array}$ & Other & Individual & $12(50 \mathrm{~min})$ & 12 & $\begin{array}{l}\text { IS: FPI } \\
\text { D: BDI } \\
\text { HAM-D }\end{array}$ & - & $3(7)$ \\
\hline Matthiesen et $a l^{74}$ & Denmark & $\begin{array}{l}\text { I: } 42(15) \\
\text { C: } 40(16)\end{array}$ & $\mathrm{RCT}$ & $\begin{array}{l}\text { Expressive writing } \\
\text { intervention }\end{array}$ & Other & Individual & $3(20 \mathrm{~min})$ & 1 & IS: COMPI & - & $4(8)$ \\
\hline $\begin{array}{l}\text { Mosalanejad } \\
\text { et } a l^{7576}\end{array}$ & Iran & $\begin{array}{l}\text { I: } 32(32) \\
\text { C: } 33(33)\end{array}$ & $\mathrm{RCT}$ & $\begin{array}{l}\text { Cognitive-behavioural } \\
\text { treatment }\end{array}$ & CBT & Group & $12(2 \mathrm{~h})$ & 12 & $\begin{array}{l}\text { D: DASS } \\
\text { A: DASS }\end{array}$ & - & $1(4)$ \\
\hline $\begin{array}{l}\text { Mosalanejad } \\
\text { et } a l^{75} 76\end{array}$ & Iran & $\begin{array}{l}\text { I: } 16(16) \\
\text { C: } 15(15)\end{array}$ & NRCT & CBT & CBT & Group & $15(1.5 h)$ & 16 & $\begin{array}{l}\text { D: DASS } \\
\text { A: DASS }\end{array}$ & - & $2(5)$ \\
\hline Catoire et $\mathrm{al}^{\mathrm{l}^{7}}$ & France & I: 50 (50) & UCT & Hypnosis & $\mathrm{MBI}$ & Individual & 4 & 1 & A: STAI & - & $4(7)$ \\
\hline Galhardo et $a^{p^{7}}$ & Portugal & $\begin{array}{l}\text { I: } 55(55) \\
\text { C: } 37(37)\end{array}$ & NRCT & $\begin{array}{l}\text { Mindfulness-based } \\
\text { programme for infertility }\end{array}$ & $\mathrm{MBI}$ & Group & $10(2 \mathrm{~h})$ & 10 & $\begin{array}{l}\text { IS: ISE } \\
\text { D: BDI } \\
\text { A: STAI }\end{array}$ & - & $1(4)$ \\
\hline Vizheh et $a^{\rho^{3}}$ & Iran & $\begin{array}{l}\text { I: } 86(86)(50 \%) \\
\text { C: } 94(86)(54.7 \%)\end{array}$ & $\mathrm{RCT}$ & Marital counselling & Other & Group & 3 (1.5hrs) & 3 & MF: MSQ & - & $4(8)$ \\
\hline
\end{tabular}

*Self-reported intervention type.

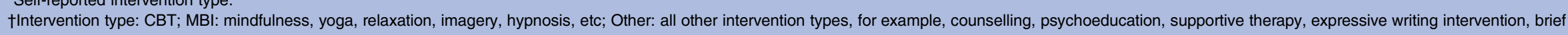
therapy, emotion and problem focused therapy, and narrative therapy.

fOutcome measures: Infertility stress: CIQ, the Coping with Infertility Questionnaire; COMPI, the Copenhagen Multi-centre Psychosocial Infertility problem stress scale; FPI, Fertility Problem Index; IDS, Infertility Distress Scale; ISD, Infertility-Specific Distress and Well-being; ISE, Infertility Self-efficacy Scale; ISS, the Infertility and Strain Scale-Depression: BDI, the Beck Depression Inventory; CES-D, the Center for Epidemiologic

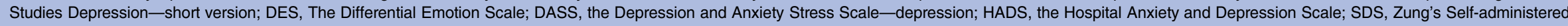
Depression Scale-Anxiety. BAI, the Beck Anxiety Inventory; Cattell, Cattell Anxiety Inventory; DASS, the Depression and Anxiety Stress Scale-anxiety; HAM-D, Hamilton Depression Rating Scale-subscale anxiety;

STAI, Spielberger State-Trait Anxiety Inventory-Marital function: C-KMS, Kansas Marital Satisfaction Scale-Chinese version RDAS (Revised Dyadic Adjustment Scale)—dyadic cohesion subscale; MSQ, Marital Satisfaction Questionnaire.

§Pregnancy is defined as a clinical pregnancy when the heartbeat of the fetal sac is evident in the uterus with an ultrasound scan.

ๆ Jadad range $0-5$ an assessment tool rating the quality and methodology of the studies included ${ }^{37}$ and the modified Jadad range $0-11$ (total score) included additional points for: inclusion of a control group, pre-post data, blinding of participants or researchers, use of standardised and reliable outcome measures and report of pre-post correlations.

CBT, cognitive-behavioural therapy; IVF, in vitro fertilisation; MBI, mind/body intervention; NRCT, non-randomised controlled trial; RCT, randomised controlled trial; UCT, uncontrolled trial (pre-post). 
compared were generally associated with smaller ES (Slope $=-0.02)$, but the association did not reach statistical significance $(\mathrm{p}=0.268)$.

\section{Quality ratings}

All included studies were methodologically assessed with the original Jadad scale and the additional methodological criteria. The original Jadad scores ranged from 0 to 4 with a mean of 2.28 (SD 1.36), and the modified total quality scores ranged from 1 to 10 with a mean of 5.36 (SD 2.05). The main methodological issue was that only very few studies attempted to blind or mask the intervention conditions to either patients or researchers. The quality ratings for each criterion for each study and total scores are shown in table 2.

\section{Effects of psychosocial intervention}

The results of the meta-analyses are shown in table 3 .

\section{Pregnancy rates}

A statistically significant and robust ES $(\mathrm{RR}=2.01)$ was found for the 10 studies which had investigated effects of psychosocial intervention on clinical pregnancy rates, with the chance of becoming pregnant being doubled in the intervention group. Adjusting for possible publication bias, the RR was somewhat lower (1.57). A forest plot of the effects of psychological intervention on pregnancy outcomes is shown in figure 2.

\section{Combined psychological outcomes}

Combining the ES of the 35 studies which had included one or more psychological outcomes revealed a statistically significant, robust, ${ }^{51}$ medium $^{39}$ ES $(g=0.59)$. The results indicated possible publication bias (skewed funnel plot, Egger's test $(p<0.05))$ in favour of larger published ES. When imputing missing ES, ${ }^{52}$ the resulting adjusted pooled ES was smaller (0.31) but remained statistically significant. Taking gender into consideration, the ES (0.51) remained statistically significant for women, still suggesting a robust effect. The ES was smaller for men (0.34) and did not reach statistical significance. A forest plot of the effects of psychological intervention on the combined psychological outcomes is shown in figure 3 .

\section{Infertility-related distress}

Only 10 studies had included infertility-related distress as an outcome. Small ES were found for women and men combined (0.24) and women alone (0.37) and did not reach statistical significance.

\section{Depression}

Twenty-one studies had assessed depressive symptoms. A statistically significant ES (1.00) was found for women and men combined. However, when adjusting for possible publication bias, the results changed dramatically to a small, non-significant ES of 0.31. Similar results were found for women alone with a statistically significant ES of 0.73 , which was reduced to a nonsignificant 0.29 after adjusting for possible publication bias. For men alone, the ES (0.13) did not reach statistical significance.

\section{State anxiety}

Twenty-five studies had included state anxiety as an outcome. A statistically significant, robust medium ES (0.51) was found for women and men combined. Adjusting for possible publication bias led to a smaller but statistically significant ES (0.31). For women, the ES of 0.53 was statistically significant, but smaller $(0.32)$ and non-significant when adjusting for publication bias. For men only, the analysis produced a small, non-significant ES of 0.32 .

\section{Marital function}

Only five studies ( $\mathrm{N}=633$ ) had included measures of marital function, but only very small (ES 0.09-0.08), non-significant effects were found.

\section{Possible moderators}

As the $Q$ statistics were generally statistically significant $(\mathrm{p}<0.10)$ and the $\mathrm{I}^{2}$ statistic indicated low-to-medium heterogeneity, when a sufficient number of studies were available for each analysis, we explored possible sources of heterogeneity and analysed whether the ES for pregnancy and combined psychological outcomes varied according to between-study differences in study design and intervention characteristics (type and format). The results are shown in table 3 .

\section{Study design}

The ES found for pregnancy outcomes were statistically significant for RCTs $(R R=1.7)$ and NRCTs (2.8), with the ES for NRCTs being considerably smaller (1.9) when adjusting for publication bias. The difference did not reach statistical significance. For psychological outcomes, statistically significant results were found for RCTs $(\mathrm{g}=0.70)$ and UCTs (0.55), but not for NRCTs (0.28) When adjusting for publication bias, the ES for RCTs was considerably reduced $(0.26)$. Furthermore, betweengroup differences did not reach statistical significance.

\section{Intervention type}

The number of studies for each intervention type was insufficient to explore differences in pregnancy outcomes. For the combined psychological outcomes, statistically significant and, as indicated by the large fail-safe numbers, robust effects were found for all three intervention categories with the largest ES found for CBT ( $\mathrm{g}=0.84$ ), followed by MBI (0.61) and other intervention types $(0.50)$. The between-group differences, did not reach statistical significance. Furthermore, the results suggested the possibility of publication bias, and when adjusting for publication bias, all three ES were reduced from medium to small. 


\begin{tabular}{|c|c|c|c|c|c|c|c|c|c|c|c|c|c|c|c|}
\hline \multirow[b]{2}{*}{ Study } & \multicolumn{7}{|l|}{ Jadad criteria } & \multicolumn{6}{|c|}{ Additional criteria } & \multirow[b]{2}{*}{$\begin{array}{l}\text { Jadad } \\
\text { Jadad } \\
\text { scores }\end{array}$} & \multirow[b]{2}{*}{$\begin{array}{l}\text { Total } \\
\text { Total } \\
\text { scores }\end{array}$} \\
\hline & $\begin{array}{l} \\
\text { Randomised }\end{array}$ & $\begin{array}{l}2 \\
\text { Double } \\
\text { blind }\end{array}$ & $\begin{array}{l}3 \\
\text { Withdrawals } \\
\text { and } \\
\text { dropouts }\end{array}$ & $\begin{array}{l}4 \\
\text { Randomisation } \\
\text { (evaluation) }\end{array}$ & $\begin{array}{l}5 \\
\begin{array}{l}\text { Blinding } \\
\text { (evaluation) }\end{array}\end{array}$ & $\begin{array}{l}6 \\
\text { Randomisation } \\
\text { (evaluation) }\end{array}$ & $\begin{array}{l}7 \\
\text { Blinding } \\
\text { (evaluation) }\end{array}$ & $\begin{array}{l}\text { a } \\
\text { Control } \\
\text { group }\end{array}$ & $\begin{array}{l}\text { b } \\
\text { Preassessment } \\
\text { and } \\
\text { postassessment }\end{array}$ & $\begin{array}{l}\text { c } \\
\begin{array}{l}\text { Blinding } \\
\text { (patients) }\end{array}\end{array}$ & $\begin{array}{l}\text { d } \\
\text { Blinding } \\
\text { (researchers) }\end{array}$ & $\begin{array}{l}\text { e } \\
\text { Standardised } \\
\text { and reliable } \\
\text { outcome }\end{array}$ & $\begin{array}{l}f \\
\text { Pre-post } \\
\text { correlation }\end{array}$ & & \\
\hline O'Moore et $a l^{79}$ & 0 & 0 & 1 & 0 & 0 & 0 & 0 & 1 & 1 & 0 & 0 & 1 & 0 & 1 & 4 \\
\hline Lukse $^{62}$ & 0 & 0 & 0 & 0 & 0 & 0 & 0 & 0 & 1 & 0 & 0 & 1 & 1 & 0 & 3 \\
\hline $\begin{array}{l}\text { Sarrel and } \\
\text { DeCherney }\end{array}$ & 0 & 0 & 0 & 0 & 0 & 0 & 0 & 1 & 0 & 0 & 0 & 0 & 0 & 0 & 1 \\
\hline Domar et $a^{63}$ & 0 & 0 & 0 & 0 & 0 & 0 & 0 & 0 & 1 & 0 & 0 & 1 & 1 & 0 & 3 \\
\hline Domar et $a^{\beta^{4}}$ & 0 & 0 & 1 & 0 & 0 & 0 & 0 & 0 & 1 & 0 & 0 & 1 & 0 & 1 & 3 \\
\hline Galletly et $a^{\beta 2}$ & 0 & 0 & 1 & 0 & 0 & 0 & 0 & 0 & 1 & 0 & 0 & 1 & 0 & 1 & 3 \\
\hline McQueeney et $a^{\beta 0}$ & 1 & 0 & 0 & 1 & 1 & 0 & 0 & 1 & 1 & 1 & 0 & 1 & 0 & 3 & 7 \\
\hline $\begin{array}{l}\text { Tuschen-Caffier } \\
\text { et } a f^{41}\end{array}$ & 0 & 0 & 1 & 0 & 0 & 0 & 0 & 1 & 1 & 0 & 0 & 1 & 0 & 1 & 4 \\
\hline Domar et al ${ }^{7895}$ & 1 & 1 & 1 & 1 & 0 & 0 & 0 & 1 & 1 & 1 & 1 & 1 & 1 & 4 & 10 \\
\hline Terzioglu $^{91}$ & 1 & 0 & 1 & 0 & 0 & 0 & 0 & 1 & 1 & 0 & 0 & 1 & 0 & 2 & 5 \\
\hline Hosaka et $a^{\beta^{7}}$ & 1 & 0 & 1 & 1 & 0 & 0 & 0 & 1 & 1 & 0 & 0 & 1 & 0 & 3 & 6 \\
\hline $\begin{array}{l}\text { McNaughton-Casill } \\
\text { et a } a^{6 *}\end{array}$ & 1 & 0 & 1 & 0 & 0 & 0 & 0 & 1 & 1 & 0 & 0 & 1 & 0 & 2 & 5 \\
\hline Emery et $a^{\beta 5}$ & 1 & 0 & 1 & 1 & 0 & 0 & 0 & 1 & 1 & 0 & 0 & 1 & 0 & 3 & 6 \\
\hline $\operatorname{Lee}^{66}$ & 1 & 0 & 0 & 0 & 0 & 0 & 0 & 1 & 1 & 0 & 0 & 1 & 0 & 1 & 4 \\
\hline De Klerk et $a^{\beta^{2}}$ & 1 & 0 & 1 & 1 & 0 & 0 & 0 & 1 & 1 & 0 & 0 & 1 & 0 & 3 & 6 \\
\hline Schmidt et $a^{\tilde{p}^{9}}$ & 0 & 0 & 1 & 0 & 0 & 0 & 0 & 1 & 1 & 0 & 0 & 1 & 0 & 1 & 4 \\
\hline Chan et $a^{\beta^{7}}$ & 1 & 0 & 1 & 1 & 0 & 0 & 0 & 1 & 1 & 0 & 0 & 1 & 1 & 3 & 7 \\
\hline Levitas et $a^{\beta 8}$ & 0 & 0 & 0 & 0 & 0 & 0 & 0 & 1 & 0 & 0 & 0 & 0 & 0 & 0 & 1 \\
\hline Nilforooshan et $a^{58}$ & 1 & 0 & 1 & 0 & 0 & 0 & 0 & 1 & 1 & 0 & 0 & 1 & 1 & 2 & 6 \\
\hline Tuil et $a^{\beta 8}$ & 1 & 0 & 1 & 1 & 0 & 0 & 0 & 1 & 1 & 0 & 0 & 1 & 0 & 3 & 6 \\
\hline Cousineau et $a^{\beta 3}$ & 1 & 0 & 1 & 1 & 1 & 0 & 0 & 1 & 1 & 1 & 0 & 1 & 0 & 4 & 8 \\
\hline Faramarzi et $a^{61}$ & 1 & 0 & 1 & 0 & 0 & 0 & 0 & 1 & 1 & 0 & 0 & 1 & 1 & 2 & 6 \\
\hline $\begin{array}{l}\text { Lancastle and } \\
\text { Boivin }^{69}\end{array}$ & 1 & 0 & 1 & 1 & 1 & 0 & 0 & 1 & 1 & 1 & 0 & 1 & 0 & 4 & 8 \\
\hline Noorbala et $a^{\beta 4 *}$ & 1 & 0 & 0 & 1 & 1 & 0 & 0 & 1 & 1 & 0 & 0 & 1 & 0 & 3 & 6 \\
\hline Mori $^{70}$ & 1 & 0 & 1 & 1 & 1 & 0 & 0 & 1 & 1 & 1 & 0 & 1 & 0 & 4 & 8 \\
\hline $\begin{array}{l}\text { Panagopoulou } \\
\text { et } a^{7^{1}}\end{array}$ & 1 & 0 & 1 & 0 & 1 & 0 & 0 & 1 & 1 & 1 & 0 & 1 & 0 & 3 & 7 \\
\hline Haemmerli et $\mathrm{al}^{72}$ & 1 & 0 & 1 & 1 & 0 & 0 & 0 & 1 & 1 & 0 & 0 & 1 & 0 & 3 & 6 \\
\hline Sexton et $a^{35}$ & 1 & 0 & 1 & 1 & 0 & 0 & 0 & 1 & 1 & 0 & 0 & 1 & 0 & 3 & 6 \\
\hline Domar et $a^{\beta 9}$ & 1 & 0 & 1 & 1 & 1 & 0 & 0 & 1 & 0 & 0 & 1 & 0 & 0 & 4 & 6 \\
\hline $\begin{array}{l}\text { Hughes and de } \\
\text { Silva }^{73}\end{array}$ & 0 & 0 & 0 & 0 & 0 & 0 & 0 & 0 & 1 & 0 & 0 & 1 & 0 & 0 & 2 \\
\hline Chan et $a \beta^{4}$ & 1 & 0 & 1 & 1 & 0 & 0 & 0 & 1 & 1 & 0 & 0 & 1 & 0 & 3 & 6 \\
\hline Gorayeb et $a^{00}$ & 1 & 0 & 1 & 1 & 0 & 0 & 0 & 1 & 0 & 0 & 0 & 0 & 0 & 3 & 4 \\
\hline Koszycki et al ${ }^{\beta 1}$ & 1 & 0 & 1 & 1 & 0 & 0 & 0 & 1 & 1 & 1 & 0 & 1 & 0 & 3 & 7 \\
\hline Matthiesen et al ${ }^{74}$ & 1 & 0 & 1 & 1 & 1 & 0 & 0 & 1 & 1 & 1 & 0 & 1 & 0 & 4 & 8 \\
\hline $\begin{array}{l}\text { Mosalanejad } \\
\text { et } a l^{75} 76\end{array}$ & 1 & 0 & 0 & 0 & 0 & 0 & 0 & 1 & 1 & 0 & 0 & 1 & 0 & 1 & 4 \\
\hline $\begin{array}{l}\text { Mosalanejad } \\
\text { et } a l^{75} 76\end{array}$ & 0 & 0 & 1 & 0 & 0 & 0 & 0 & 1 & 1 & 0 & 0 & 1 & 0 & 1 & 4 \\
\hline Catoire et $\mathrm{al}^{77 \star}$ & 0 & 0 & 0 & 0 & 0 & 0 & 0 & 0 & 1 & 0 & 1 & 1 & 0 & 0 & 3 \\
\hline Galhardo et $a^{\rho^{7}}$ & 0 & 0 & 1 & 0 & 0 & 0 & 0 & 1 & 1 & 0 & 0 & 1 & 0 & 1 & 4 \\
\hline Vizheh et $a^{\beta^{3}}$ & 1 & 0 & 1 & 1 & 1 & 0 & 0 & 1 & 1 & 0 & 1 & 1 & 0 & 4 & 8 \\
\hline
\end{tabular}

Criteria 1-7 in bold font are the original Jadad scores, a-f are the additional criteria. (1) Was the study described as randomised; (2) Was the study described as double blind; (3) Was there a description of withdrawals and dropouts; (4) The method of randomisation was described, and appropriate; (5) The method of blinding was described, and appropriate; (6) The method of randomisation was described, but inappropriate; (7) The method of blinding was described,

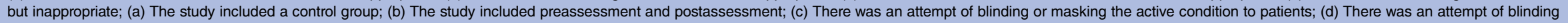
the researchers; (e) The study used standardised and reliable outcome measures and ( $\mathrm{f}$ ) The study reported pre-post correlation.

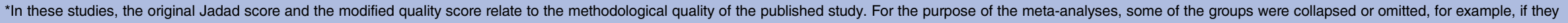




\begin{tabular}{|c|c|c|c|c|c|c|c|c|c|c|c|}
\hline & \multirow{2}{*}{$\begin{array}{l}\text { Sample size } \\
\mathrm{K}\end{array}$} & \multirow[b]{2}{*}{$\mathbf{N}$} & \multicolumn{2}{|c|}{ Heterogeneity* } & \multirow[b]{2}{*}{ p Value } & \multirow[b]{2}{*}{$I^{2}$} & \multicolumn{2}{|l|}{ Global ES } & \multirow[b]{2}{*}{$\mathrm{p}$ Value } & \multirow{2}{*}{$\begin{array}{l}\text { Fail-safe } \\
\text { numbers } \neq\end{array}$} & \multirow[b]{2}{*}{ Criterion§ } \\
\hline & & & $\overline{\mathbf{Q}}$ & df & & & Hedges g† & $95 \% \mathrm{Cl}$ & & & \\
\hline \multicolumn{12}{|l|}{ Main effects } \\
\hline \multicolumn{12}{|l|}{ Pregnancy } \\
\hline Pregnancy, women & 10 & 1324 & 22.0 & 9 & 0.009 & 59.0 & $2.01(\mathrm{RR})$ & 1.48 to 2.73 & $<0.001$ & 130 & 60 \\
\hline Adjusted for publication bias & (13) & - & - & - & - & - & $1.57(R R)$ & 1.10 to 2.25 & $<0.05$ & - & - \\
\hline Psych. combined, women+men & 35 & 2746 & 259.2 & 34 & $<0.001$ & 86.9 & 0.59 & 0.38 to 0.80 & $<0.001$ & 1552 & 185 \\
\hline Adjusted for publication bias & (42) & - & - & - & - & - & 0.31 & 0.07 to 0.56 & $<0.05$ & - & - \\
\hline Psych. combined, women & 28 & 2076 & 130.8 & 27 & $<0.001$ & 76.4 & 0.51 & 0.32 to 0.70 & $<0.001$ & 798 & 150 \\
\hline Adjusted for publication bias & (34) & - & - & - & - & - & 0.30 & 0.09 to 0.51 & $<0.05$ & - & - \\
\hline Psych. combined, men & 7 & 347 & 8.9 & 6 & 0.178 & 32.8 & 0.34 & 0.08 to 0.59 & 0.010 & 12 & 45 \\
\hline Between-group ${ }^{\star \star}$ (women vs men) & 35 & 2110 & 1.2 & 1 & NS & - & - & - & - & - & - \\
\hline \multicolumn{12}{|l|}{ Infertility distress } \\
\hline Infertility distress, women+men & 10 & 615 & 21.4 & 9 & 0.01 & 58.0 & 0.24 & -0.02 to 0.50 & NS & - & - \\
\hline Infertility distress, women & 6 & 371 & 17.8 & 5 & 0.003 & 71.8 & 0.37 & -0.06 to 0.79 & NS & - & - \\
\hline \multicolumn{12}{|l|}{ Depressive symptoms } \\
\hline Depression symptom, women+men & 21 & 1558 & 367.5 & 20 & $<0.001$ & 94.6 & 1.00 & 0.54 to 1.45 & $<0.001$ & 1022 & 115 \\
\hline Adjusted for publication bias & (25) 9 & - & - & - & - & - & 0.31 & -0.20 to 0.84 & NS & - & - \\
\hline Depressive symptom, women & 17 & 992 & 107.7 & 16 & $<0.001$ & 85.1 & 0.73 & 0.41 to 1.06 & $<0.001$ & 393 & 95 \\
\hline Adjusted for publication bias & (23) 9 & - & - & - & - & - & 0.29 & -0.07 to 0.65 & NS & - & - \\
\hline Depressive symptom, men & 5 & 243 & 1.9 & 4 & 0.749 & 0.00 & 0.13 & -0.11 to 0.37 & NS & - & - \\
\hline Between-group ${ }^{\star \star}$ (women vs men) & 22 & 1235 & 8.5 & 1 & $<0.004$ & - & - & - & - & - & - \\
\hline \multicolumn{12}{|l|}{ Anxiety } \\
\hline Anxiety, women+men & 25 & 2159 & 144.4 & 24 & $<0.001$ & 83.4 & 0.51 & 0.31 to 0.71 & $<0.001$ & 760 & 135 \\
\hline Adjusted for publication bias & (29) & - & - & - & - & - & 0.31 & 0.07 to 0.54 & $<0.05$ & - & - \\
\hline Anxiety, women & 23 & 1737 & 114.3 & 22 & $<0.001$ & 80.8 & 0.53 & 0.32 to 0.73 & $<0.001$ & 631 & 125 \\
\hline Adjusted for publication bias & (27) 9 & - & - & - & - & - & 0.32 & 0.08 to 0.57 & $<0.05$ & - & - \\
\hline Anxiety, men & 5 & 246 & 8.7 & 4 & 0.070 & 53.8 & 0.32 & -0.04 to 0.67 & NS & - & - \\
\hline Between-group ${ }^{\star \star}$ (women vs men) & 28 & 1983 & 1.0 & 1 & NS & - & - & - & - & - & - \\
\hline \multicolumn{12}{|l|}{ Marital function } \\
\hline Marital function, women+men & 5 & 633 & 14.6 & 4 & 0.006 & 72.6 & 0.09 & -0.23 to 0.41 & NS & - & - \\
\hline Marital function, women & 4 & 587 & 14.5 & 3 & 0.002 & 79.3 & 0.08 & -0.30 to 0.46 & NS & - & - \\
\hline \multicolumn{12}{|l|}{ Moderator analyses } \\
\hline \multicolumn{12}{|l|}{ Pregnancy (women) } \\
\hline \multicolumn{12}{|l|}{ Study designt† } \\
\hline RCT & 6 & 856 & 10.8 & 5 & 0.057 & 53.5 & $1.67(\mathrm{RR})$ & 1.17 to 2.40 & $<0.05$ & 22 & 40 \\
\hline NRCT & 4 & 468 & 7.9 & 3 & 0.048 & 62.1 & $2.80(\mathrm{RR})$ & 1.55 to 5.06 & $<0.001$ & 31 & 30 \\
\hline Adjusted for publication bias & (6) 9 & - & - & - & - & - & $1.93(R R)$ & 1.07 to 3.49 & $<0.05$ & - & - \\
\hline Between-group ${ }^{\star \star}$ & 10 & 1324 & 2.1 & 1 & NS & - & - & - & - & - & - \\
\hline \multicolumn{12}{|l|}{ Intervention format } \\
\hline Group & 5 & 691 & 10.9 & 4 & 0.027 & 63.4 & $2.03(\mathrm{RR})$ & 1.29 to 3.20 & $<0.01$ & 28 & 35 \\
\hline Individual & 4 & 433 & 2.2 & 3 & 0.531 & 0.0 & 1.65 (RR) & 1.26 to 2.17 & $<0.001$ & 8 & 30 \\
\hline Between-group ${ }^{\star \star}$ & 9 & 1124 & 0.5 & 1 & NS & - & - & - & - & - & - \\
\hline
\end{tabular}




\begin{tabular}{|c|c|c|c|c|c|c|c|c|c|c|c|}
\hline & \multirow{2}{*}{$\begin{array}{l}\text { Sample size } \\
\mathbf{K}\end{array}$} & \multirow[b]{2}{*}{$\mathbf{N}$} & \multicolumn{2}{|c|}{ Heterogeneity* } & \multirow[b]{2}{*}{ p Value } & \multirow[b]{2}{*}{$1^{2}$} & \multicolumn{2}{|l|}{ Global ES } & \multirow[b]{2}{*}{ p Value } & \multirow{2}{*}{$\begin{array}{l}\text { Fail-safe } \\
\text { numbers } \neq\end{array}$} & \multirow[b]{2}{*}{ Criterion§ } \\
\hline & & & $\overline{\mathbf{Q}}$ & df & & & Hedges g† & $95 \% \mathrm{Cl}$ & & & \\
\hline \multicolumn{12}{|c|}{ Psychological outcomes combined (women+men) } \\
\hline \multicolumn{12}{|l|}{ Study design†† } \\
\hline RCT & 20 & 2185 & 232.4 & 19 & $<0.001$ & 91.8 & 0.70 & 0.36 to 1.03 & $<0.001$ & 642 & 110 \\
\hline Adjusted for publication bias & $(24) 9$ & - & - & - & - & - & 0.26 & -0.10 to 0.68 & NS & - & - \\
\hline NRCT & 8 & 450 & 14.9 & 7 & 0.037 & 53.1 & 0.28 & -0.00 to 0.57 & NS & - & - \\
\hline UCT & 7 & 215 & 6.0 & 6 & 0.424 & 0.0 & 0.55 & 0.40 to 0.70 & $<0.001$ & 90 & 45 \\
\hline Adjusted for publication bias & $(10) 9$ & - & - & - & - & - & 0.51 & 0.36 to 0.66 & $<0.05$ & - & - \\
\hline Between-group*** & 35 & 2850 & 3.9 & 2 & NS & - & - & - & - & - & - \\
\hline \multicolumn{12}{|l|}{ Intervention types } \\
\hline CBT & 7 & 475 & 39.0 & 6 & $<0.001$ & 84.6 & 0.84 & 0.33 to 1.35 & 0.001 & 107 & 45 \\
\hline Adjusted for publication bias & $(10) 9$ & - & - & - & - & - & 0.37 & -0.19 to 0.93 & NS & - & - \\
\hline $\mathrm{MBI}$ & 9 & 841 & 57.7 & 8 & $<0.001$ & 86.1 & 0.61 & 0.17 to 0.65 & $<0.001$ & 158 & 55 \\
\hline Adjusted for publication bias & $(10) 9$ & - & - & - & - & - & 0.42 & 0.01 to 0.84 & $<0.05$ & - & - \\
\hline Other & 19 & 1430 & 149.2 & 9 & $<0.001$ & 87.9 & 0.50 & 0.18 to 0.81 & 0.002 & 246 & 105 \\
\hline Adjusted for publication bias & $(24) \eta$ & - & - & - & - & - & 0.17 & -0.20 to 0.54 & NS & - & - \\
\hline Between-group*** & 35 & 2746 & 1.3 & 2 & NS & - & - & - & - & - & \\
\hline \multicolumn{12}{|l|}{ Intervention format } \\
\hline Group & 20 & 1484 & 87.2 & 19 & $<0.001$ & 78.2 & 0.76 & 0.55 to 0.98 & $<0.001$ & 959 & 110 \\
\hline Adjusted for publication bias & $(26) 9$ & - & - & - & - & - & 0.50 & 0.25 to 0.75 & $<0.05$ & - & - \\
\hline Individual & 9 & 834 & 17.7 & 8 & 0.023 & 54.9 & 0.13 & -0.08 to 0.35 & NS & - & - \\
\hline Couples & 3 & 284 & 92.3 & 2 & $<0.001$ & 97.8 & 1.07 & -1.02 to 3.16 & NS & - & - \\
\hline Online & 3 & 248 & 1.2 & 2 & 0.541 & 0.00 & 0.03 & -0.22 to 0.28 & NS & - & - \\
\hline Between-group** & 35 & 2850 & 24.5 & 3 & $<0.001$ & - & - & - & - & - & \\
\hline \multicolumn{12}{|c|}{ 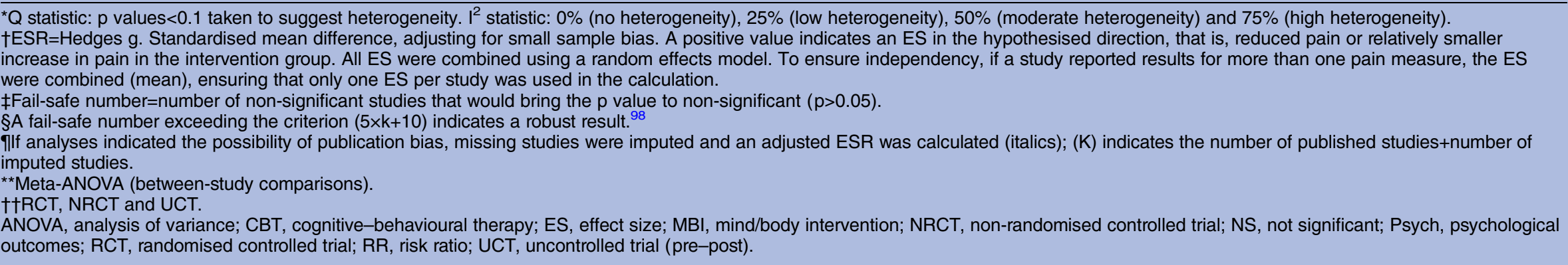 } \\
\hline
\end{tabular}


Pregnancy rates

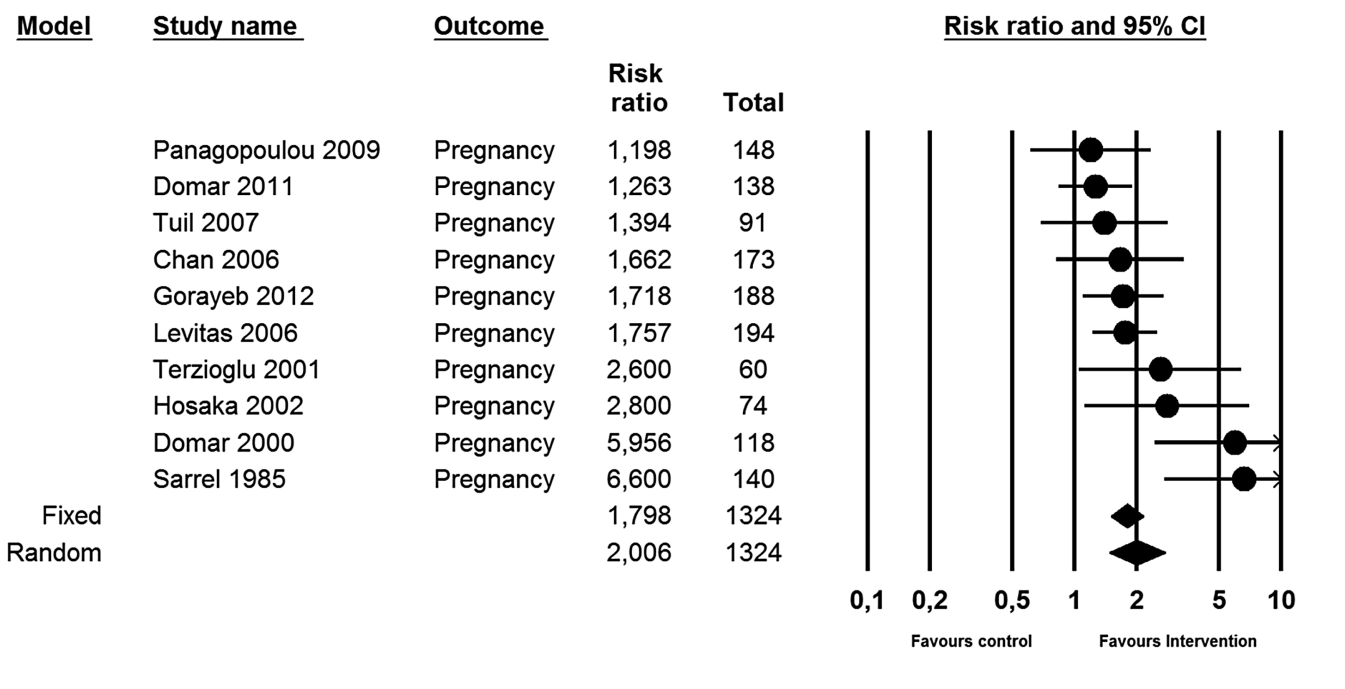

Figure 2 Effects of psychosocial intervention on pregnancy rates.

\section{Combined psychological outcomes}

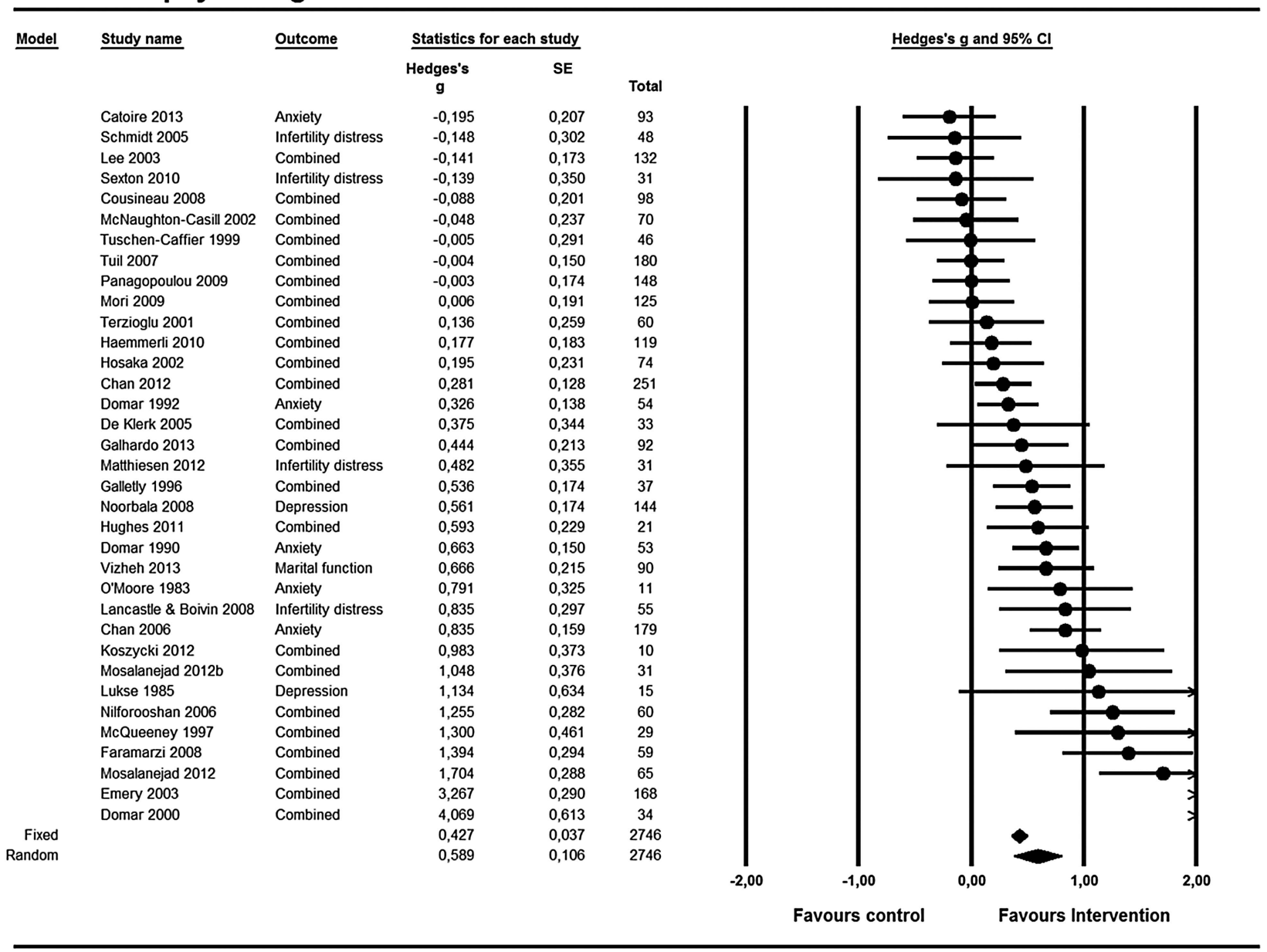

Figure 3 Effects of psychosocial intervention on combined psychological outcomes. 
Intervention format

For pregnancy outcomes, the number of studies was sufficient for group and individual formats. Both formats yielded statistically significant ES (RR 2.03 and 1.65), and the between-group difference did not reach statistical significance. For the combined psychological outcomes, a statistically significant effect was found for the Group format $(\mathrm{g}=0.76 ; \mathrm{p}<0.001)$. The ES for intervention formats such as individual, couples and online did not reach statistical significance. The overall between-group difference for intervention formats was statistically significant $(\mathrm{p}<0.001)$.

\section{Other study characteristics}

The possible moderating influence of the continuously assessed study characteristics of mean age, intervention duration, number or sessions, and study quality (modified quality scores) were analysed with meta-regression. As seen in table 4, no significant effects were found for any of the moderators for either pregnancy or the combined psychological outcomes. A total of six studies had examined the effects on pregnancy and anxiety. When examining the possible role of anxiety reduction as a mediator of the effect on pregnancy outcome with meta-regression, a statistically significant association was found between the ES for anxiety and pregnancy, indicating that the greater the reduction in anxiety, the greater the likelihood of achieving pregnancy (see table 4).

\section{DISCUSSION}

\section{Primary findings}

Our meta-analysis of the available evidence suggests that women who receive some form of psychological intervention are approximately twice as likely to become pregnant when compared with controls receiving standardised care or active control intervention. Although the results of the 10 currently available studies taken together appeared robust, there were some indications of publication bias in favour of studies with larger positive ES. It should also be noted that the precision of the
ES estimate is limited, with possible RRs ranging from approximately 1.5 to 2.7. Furthermore, although the between-group difference did not reach statistical significance when disregarding the possibility of publication bias, NRCTs yielded greater effects (RR 2.8 (95\% CI 1.55 to 5.06)) than RCTs (RR 1.7 (95\% CI 1.17 to 2.40)). Compared with other types of interventions that historically have been introduced to improve pregnancy rates in ART (improved culture media, new hormone stimulation regimens, etc), even an effect corresponding to the lower limit of the CI is substantial. While the results could be considered surprising, the available data do not provide any clear-cut reasons to reject this finding, which is further supported by the results of the meta-regression showing that larger reductions in anxiety were associated with improved pregnancy outcomes. With respect to the psychological outcomes currently reported in the literature, the results suggest that psychological intervention could be effective in reducing anxiety (25 studies) as well as depressive symptoms (21 studies) with the effects corresponding to medium and large ES (0.5 and 1.0). As seen for pregnancy outcomes, there were indications of publication bias in the direction of larger positive effects, and adjusting for publication bias resulted in a considerably smaller, statistically non-significant, ES for depressive symptoms. The pooled results did not reach statistical significance for the 10 studies which had investigated effects on infertilityrelated distress and the 5 studies which had included measures of marital function.

\section{Comparing with results of previous reviews}

The present review included 39 studies with a total of 3401 women (3064) and men (347). The participants received various psychosocial interventions lasting from 1 week to 6 months, including CBT, emotional disclosure, psychoeducation and MBIs. The present review evaluates almost twice the number of studies included in the most recent previous review, ${ }^{31}$ which reported mixed results of the efficacy of psychosocial intervention.

Table 4 Results of meta-regression analyses

\begin{tabular}{|c|c|c|c|c|c|}
\hline Dependent variable & Independent variable & $\mathbf{K}$ & $\boldsymbol{\beta}^{*}$ & $95 \% \mathrm{Cl}$ & p Value \\
\hline \multirow[t]{5}{*}{ Pregnancy } & ES-anxiety & 6 & 0.19 & 0.06 to 0.31 & 0.004 \\
\hline & Mean age & 9 & -0.05 & -0.19 to 0.10 & 0.534 \\
\hline & Intervention duration & 9 & 0.01 & -0.03 to 0.06 & 0.669 \\
\hline & Number of sessions & 9 & -0.00 & -0.08 to 1.07 & 0.922 \\
\hline & Study quality (quality scores) $\dagger$ & 10 & -0.02 & -0.09 to 0.04 & 0.477 \\
\hline \multirow[t]{4}{*}{ Psych. combined } & Mean age & 32 & -0.05 & -0.12 to -0.02 & 0.214 \\
\hline & Intervention duration & 32 & 0.01 & -0.02 to 0.04 & 0.518 \\
\hline & Number of sessions & 27 & 0.03 & -0.01 to 0.07 & 0.150 \\
\hline & Study quality (quality scores)†‡ & 35 & -0.02 & -0.06 to 0.02 & 0.415 \\
\hline
\end{tabular}


Whereas the former review found no evidence for attenuating distress, there was promising support of psychological intervention increasing pregnancy chances for women not receiving ART. ${ }^{31}$ In line with the second review from $2005,{ }^{30}$ we found more credible results for group intervention than for other formats, for example, online, individual and couples interventions. ${ }^{30}$ The first review published in 2003 also highlighted group interventions as more effective, especially if the interventions emphasised education and skills training, such as relaxation. Our results concurred with these earlier observations, suggesting that interventions delivered in groups may be more effective in reducing distress. Moreover, although the comparison did not reach statistical significance, prior to adjusting for publication bias, the intervention type of CBT appeared to be more effective than MBI and other types of interventions. It should be noted that the categorisation of interventions may be somewhat ambiguous. For example, the study by Cousineau et $a l^{83}$ could have been categorised as an MBI, as the authors had provided a website that directed attention towards relaxation exercises. However, as there was no reporting of whether the participants were engaged in weekly or daily training, we chose to interpret relaxation as an optional feature, and hence the study was not categorised as an MBI. The possible ambiguity and considerable variability in interventions forced us to categorise many studies as 'other', which limits our understanding of the possible mechanisms in psychosocial interventions. Taken together, the available data do not provide a clear basis for understanding the possible differences between effects of different intervention types, and the results should be interpreted with caution. The more recently conducted studies included in the present review have contributed by increasing the size of the available data set considerably, and taken together, the currently available evidence suggests that offering psychosocial interventions may improve both the chances of pregnancy and the quality of life for infertile patients going through fertility treatment.

\section{Strengths and limitations}

Our systematic review and meta-analysis has several strengths. We conducted a comprehensive search and performed the review in accordance with the recommended guidelines. ${ }^{34}$ In order to limit the possibility of selection bias, we encouraged authors of eligible studies to elaborate on their results if the data reported were insufficient, and asked authors of papers written in a foreign language to submit their results to us in English. The included studies represented a range of different countries, have used comparable outcome measures, and provided fairly comprehensive descriptions of the interventions studied. In addition, we conducted a detailed evaluation of the methodological quality in order to detect any issues that could possible affect the accuracy of the ES calculated. While not all characteristics, in particular reproductive, could be assessed; most general methodological aspects were covered. We also explored heterogeneity and made adjustments for possible publication bias, when required.

Some limitations of the currently available data should also be noted. First, the samples investigated may not have been as homogeneous as could be wished for. A small number of infertile participants did not receive treatment with ART, and furthermore, it was not consistently reported what type of ART procedure the participants received, what phase or treatment they were in, or the causes of infertility. This information is clearly important when interpreting the outcomes, and unknown between-study and within-study between-group differences, for example, in numbers of cycles, idiopathic infertility and embryo transfer, may have influenced the results, in particular for pregnancy outcomes. However, such differences are likely to be less important in RCTs, where randomisation is expected to reduce their influence. Although the difference did not reach statistical significance, RCTs reported smaller ES for pregnancy outcomes than NRCTs, which could be interpreted as supporting the concern that infertility and treatment characteristics may have been unevenly distributed between psychological treatment arms, thus increasing the risk for misattribution of outcomes to intervention, at least for NRCTs. On the other hand, we found no statistical significant associations between study quality scores and either pregnancy or psychological outcomes, no statistically significant differences in dropout rates between intervention and control groups, and, as suggested by the large fail-safe numbers, the improvements generally appeared quite robust. A second possible limitation is the high level of heterogeneity indicated by the $Q$ and $\mathrm{I}^{2}$ statistics, and the pooled ES reported in the present review should thus be viewed as an estimate of the average expected effect across a wide range of different settings. A third issue is that the considerable dropout rates and lack of ITT analyses may have influenced the results, and it cannot be excluded that fertility-related and treatment-related factors such as non-optimal fertilisation, small number of eggs, etc, may have demotivated some participants and made them dropout of the study, while individuals who progressed through the treatment phases with more satisfactory outcomes were more likely to complete the study. Fourth, the indications of publication bias found for several results suggest the possibility of a 'file drawer problem', that is, the existence of relevant unpublished null findings, a common problem when conducting systematic reviews. Finally, owing to inconsistencies in the reporting of causes of infertility, we are unable to evaluate the possible associations between ES and causes of infertility. Although meta-analysis remains the gold standard when evaluating the current evidence within a field of research, as is often the case with systematic reviews, qualitative as well as quantitative, the overall level of the evidence reported in our review may be challenged by publication bias and the heterogeneity and methodological limitations of the available published studies. 


\section{Clinical and practical implications}

We found evidence for improvement in general psychological symptoms such as anxiety and depression, but not for infertility-specific distress. A possible explanation for the latter could be the lack of sensitivity of the infertility-related distress measures used. The questions used in these measures are directly concerned with thoughts and feelings about involuntary childlessness, and rumination about the involuntary childlessness may persist even when psychosocial intervention improves general psychological well-being. Of particular interest is the result of our meta-regression analysis of the six studies which had included both pregnancy and anxiety as outcomes showing that larger reductions in anxiety were associated with greater chances of pregnancy. Anxiety is a state of arousal, which over time is physically and mentally stressful for the individual. ${ }^{17}$ Reducing distress, anxiety in particular, may increase the physiological ability to cope with stress and advance the possibility of impregnation. We found no association between mean age and pregnancy rates outcomes, which may seem surprising, since age is the most important predictor of pregnancy outcomes of ART. ${ }^{99} 100$ However, our meta-regression was conducted for the mean age of the samples, and the mean age across study samples showed little variation (mean age 32.7; SD 2.4). The rather narrow age interval across study samples may explain an apparent lack of association between age and chance of pregnancy. Our findings also suggest that group interventions appear to be more efficacious than individual, couples or online interventions. There could be various reasons for this. First, group interventions had a longer duration (mean 9.5 weeks) and involved more sessions (8.3) than individual interventions (mean 5.3 and 4.4), and second, there is evidence of a positive impact of 'group settings', that is, the sense of community between participants, reducing the feelings of isolation or alienation and sharing with individuals in the same life situation, etc. ${ }^{101-104}$

\section{Recommendations for future research}

Despite the overall positive effects of psychosocial interventions found in the literature, our results suggest a need for further studies with more rigorous methodology, including more strict reporting of causes of infertility, the types of ART used, and which phases of treatment the participants are in. Also, most of the studies were conducted in high-income countries; it is therefore important to note that the assertions made here cannot be generalised to low-income and developing countries. There is thus a need for research in low-income or developing countries as well. Another aspect pertaining to generalisability is the challenge of comparing volunteering infertile participants in psychosocial efficacy studies with the general population of infertile individuals. The response rates in this area are moderate, and it seems important in future studies to explore and compare characteristics of dropouts and completers, as well as of non-responders and responders. Furthermore, it would be of importance to develop clinically meaningful categories of distress with the purpose of improving interventions targeted to the various types and levels of distress experienced by the participants. Psychological well-being/distress fluctuates over time during fertility treatment and a stepped care approach could be potentially valuable in this population. ${ }^{105}$ It is also possible that interventions aimed at relieving distress conducted at different phases in treatment may obtain different psychological outcome results. This calls for improved reporting and comparability of the timing of psychosocial interventions and greater precision and comparability of the timing of outcome assessments. Also needed are studies testing specific hypotheses concerning possible moderating and mediating mechanisms of the effects of interventions on distress and pregnancy outcomes. For example, which psychosocial factors do we need to target to optimise effects on distress and pregnancy rates, and which biomarkers affected by psychosocial interventions, for example, oxidative stress, inflammatory processes, can best explain the observed effects? This could assist in developing a more solid evidence base providing better guidance for patients, health professionals and policymakers about 'what works for whom' in infertile patients.

\section{CONCLUSIONS}

In conclusion, the present meta-analysis of 39 studies suggests that psychosocial interventions, in particular CBT and MBI interventions, are beneficial for reducing distress and for improving pregnancy outcomes of ART. Moreover, there is some preliminary evidence to suggest that reduction in anxiety achieved through psychological intervention may improve the chance of pregnancy. Despite the robust overall effect found, the considerable heterogeneity of the available studies with respect to methodological quality, intervention type and format still warrants caution as to the conclusions which can be drawn.

\section{Author affiliations}

${ }^{1}$ Unit for Psychooncology and Health Psychology, Department of Psychology, Aarhus University, Aarhus, Denmark

${ }^{2}$ Skejby Fertility Clinic, Aarhus University Hospital, Aarhus, Denmark ${ }^{3}$ Department of Oncology, Aarhus University Hospital, Aarhus, Denmark

Acknowledgements The authors would like to thank Gina Bay, Librarian at the Library, Department of Psychology and Behavioral Sciences, School of Business and Social Sciences, Aarhus University for valuable and tireless assistance throughout the database search process. They would also like to give their most grateful thanks to the researchers who provided them with additional data on their studies or aided them in other ways.

Contributors RZ and YF designed the protocol. YF developed search strategies and IF-V, NGS and YF performed the searches and study selection. YF and IF-V undertook data extraction and quality assessments. RZ was responsible for analysing the data. YF drafted the manuscript and IF-V, NGS, $\mathrm{HJI}$ and $\mathrm{RZ}$ contributed to the manuscript throughout the process. All authors provided inputs and were involved with the interpretation of this review. YF is the study guarantor. 
Funding YF was supported by a research grant from The Danish Agency for Science Technology and Innovation.

Competing interests None.

Provenance and peer review Not commissioned; externally peer reviewed.

Data sharing statement Extra data can be accessed via the Dryad data repository at http://datadryad.org/ with the doi:10.5061/dryad.kv50v.

Open Access This is an Open Access article distributed in accordance with the Creative Commons Attribution Non Commercial (CC BY-NC 4.0) license, which permits others to distribute, remix, adapt, build upon this work noncommercially, and license their derivative works on different terms, provided the original work is properly cited and the use is non-commercial. See: http:// creativecommons.org/licenses/by-nc/4.0/

\section{REFERENCES}

1. Hart VA. Infertility and the role of psychotherapy. Issues Ment Health Nurs 2002;23:31-41.

2. Oddens BJ, den Tonkelaar I, Nieuwenhuyse H. Psychosocial experiences in women facing fertility problems-a comparative survey. Hum Reprod 1999;14:255-61.

3. Cwikel J, Gidron Y, Sheiner E. Psychological interactions with infertility among women. Eur J Obstet Gynecol Reprod Biol 2004;117:126-31.

4. Swan SH, Elkin EP, Fenster L. The question of declining sperm density revisited: an analysis of 101 studies published 1934-1996. Environ Health Perspect 2000;108:961-6.

5. Skakkebaek NE, Jorgensen N, Main KM, et al. Is human fecundity declining? Int J Androl 2006;29:2-11.

6. Hammen C. Stress and depression. Annu Rev Clin Psychol 2005;1:293-319.

7. Benyamini Y, Gozlan M, Kokia E. Variability in the difficulties experienced by women undergoing infertility treatments. Fertil Steril 2005;83:275-83.

8. Alesi R. Infertility and its treatment-an emotional roller coaster. Aust Fam Physician 2005;34:135-8.

9. Merari D, Chetrit A, Modan B. Emotional reactions and attitudes prior to in vitro fertilization: an inter-spouse study. Psychol Health 2002;17:629-40.

10. Klonoff-Cohen $\mathrm{H}$, Chu E, Natarajan L, et al. A prospective study of stress among women undergoing in vitro fertilization or gamete intrafallopian transfer. Fertil Steril 2001;76:675-87.

11. Edelmann RJ, Connolly KJ, Bartlett H. Coping strategies and psychological adjustment of couples presenting for IVF. J Psychosom Res 1994;38:355-64.

12. Eugster A, Vingerhoets AJ. Psychological aspects of in vitro fertilization: a review. Soc Sci Med 1999;48:575-89.

13. Stanton AL, Dunkel-Schetter C, eds. Infertility: perspectives from stress and coping research. In: Psychological adjustment to infertility: an overview of conceptual approaches. The Plenum series on stress and coping. New York, NY, USA: Plenum Press, 1991:3-16.

14. Benyamini Y, Gozlan M, Kokia E. Women's and men's perceptions of infertility and their associations with psychological adjustment: a dyadic approach. Br J Health Psychol 2009;14:1-16.

15. du Plessis SS, Makker K, Desai NR, et al. Impact of oxidative stress on IVF. Expert Rev Obstet Gynecol 2008;3:539-54.

16. Agarwal A, Allamaneni SS. Role of free radicals in female reproductive diseases and assisted reproduction. Reprod Biomed Online 2004;9:338-47.

17. Tracey KJ. The inflammatory reflex. Nature 2002;420:853-9.

18. Kiecolt-Glaser JK, McGuire L, Robles TF, et al. Emotions, morbidity, and mortality: new perspectives from psychoneuroimmunology. Annu Rev Psychol 2002;53:83-107.

19. Csemiczky G, Landgren BM, Collins A. The influence of stress and state anxiety on the outcome of IVF-treatment: psychological and endocrinological assessment of Swedish women entering IVF-treatment. Acta Obstet Gynecol Scand 2000;79:113-18.

20. Stoleru S, Teglas JP, Fermanian J, et al. Psychological factors in the aetiology of infertility: a prospective cohort study. Hum Reprod 1993:8:1039-46.

21. Demyttenaere K, Nijs P, Evers-Kiebooms G, et al. Coping and the ineffectiveness of coping influence the outcome of in vitro fertilization through stress responses. Psychoneuroendocrinology 1992;17:655-65.
22. Sanders KA, Bruce NW. Psychosocial stress and treatment outcome following assisted reproductive technology. Hum Reprod 1999;14:1656-62.

23. Smeenk JM, Verhaak CM, Vingerhoets AJ, et al. Stress and outcome success in IVF: the role of self-reports and endocrine variables. Hum Reprod 2005;20:991-6.

24. Ebbesen SM, Zachariae R, Mehlsen MY, et al. Stressful life events are associated with a poor in-vitro fertilization (IVF) outcome: a prospective study. Hum Reprod 2009;24:2173-82.

25. Klonoff-Cohen $\mathrm{H}$. Female and male lifestyle habits and IVF: what is known and unknown. Hum Reprod Update 2005;11:179-203.

26. Homan GF, Davies M, Norman R. The impact of lifestyle factors on reproductive performance in the general population and those undergoing infertility treatment: a review. Hum Reprod Update 2007:13:209-23.

27. Matthiesen SM, Frederiksen $\mathrm{Y}$, Ingerslev HJ, et al. Stress, distress and outcome of assisted reproductive technology (ART) a meta-analysis. Hum Reprod 2011;26:2763-76.

28. Boivin J, Griffiths E, Venetis CA. Emotional distress in infertile women and failure of assisted reproductive technologies: metaanalysis of prospective psychosocial studies. BMJ 2011;342:d223.

29. Boivin J. A review of psychosocial interventions in infertility. Soc Sci Med 2003:57:2325-41.

30. de Liz TM, Strauss B. Differential efficacy of group and individual/ couple psychotherapy with infertile patients. Hum Reprod 2005;20:1324-32.

31. Haemmerli K, Znoj H, Barth J. The efficacy of psychological interventions for infertile patients: a meta-analysis examining mental health and pregnancy rate. Hum Reprod Update 2009;15:279-95

32. Hoffmann TC, Glasziou PP, Boutron I, et al. Better reporting of interventions: template for intervention description and replication (TIDieR) checklist and guide. BMJ 2014;348:g1687.

33. Liberati A, Altman DG, Tetzlaff J, et al. The PRISMA statement for reporting systematic reviews and meta-analyses of studies that evaluate health care interventions: explanation and elaboration PLoS Med 2009;6:e1000100.

34. Moher D, Liberati A, Tetzlaff J, et al. Preferred reporting items for systematic reviews and meta-analyses: the PRISMA statement. PLoS Med 2009;6:e1000097.

35. Higgins J, Green S, eds. Cochrane handbook for systematic reviews of interventions. Version 5.1.0 [updated March 2011]. The Cochrane Collaboration, 2011. http://www.cochrane-handbook.org

36. Gurunath S, Pandian Z, Anderson RA, et al. Defining infertility-a systematic review of prevalence studies. Hum Reprod Update 2011;17:575-88.

37. Jadad AR, Moore RA, Carroll D, et al. Assessing the quality of reports of randomized clinical trials: is blinding necessary? Control Clin Trials 1996;17:1-12.

38. Greenland S, O'rourke K. On the bias produced by quality scores in meta-analysis, and a hierarchical view of proposed solutions. Biostatistics 2001;2:463-71.

39. Cohen J. Statistical power analysis for the behavioral sciences. Hillsdale, NJ: Lawrence Erlbaum Associates, 1988.

40. Hedges L, Olkin I. Statistical methods for meta-analysis. New York: Academic Press, 1985.

41. Tuschen-Caffier B, Florin I, Krause W, et al. Cognitive-behavioral therapy for idiopathic infertile couples. Psychother Psychosom 1999;68:15-21.

42. Hozo S, Djulbegovic B, Hozo I. Estimating the mean and variance from the median, range, and the size of a sample. BMC Med Res Methodol 2005;5:13. http://www.biomedcentral.com/1471-2288/5/13.

43. Sterne JAC, Egger M, Moher D. Addressing reporting biases. John Wiley \& Sons, Ltd, 2008:297-333.

44. Poole C, Greenland S. Random-effects meta-analyses are not always conservative. Am J Epidemiol 1999;150:469-75.

45. Higgins JP, Thompson SG, Deeks JJ, et al. Measuring inconsistency in meta-analyses. BMJ 2003;327:557-60.

46. Hedges LV, Pigott TD. The power of statistical tests in meta-analysis. Psychol Methods 2001;6:203-17.

47. Ioannidis JP, Trikalinos TA. The appropriateness of asymmetry tests for publication bias in meta-analyses: a large survey. CMAJ 2007; 176:1091-6.

48. Copas J, Shi JQ. Meta-analysis, funnel plots and sensitivity analysis. Biostatistics 2000;1:247-62.

49. Deeks JJ, Macaskill P, Irwig L. The performance of tests of publication bias and other sample size effects in systematic reviews of diagnostic test accuracy was assessed. J Clin Epidemiol 2005;58:882-93.

50. Egger M, Davey Smith G, Schneider M, et al. Bias in meta-analysis detected by a simple, graphical test. BMJ 1997;315:629-34. 
51. Rosenthal R. The file drawer problem and tolerance for null results. Psychol Bull 1979;86:638-41.

52. Duval S, Tweedie R. Trim and fill: a simple funnel-plot-based method of testing and adjusting for publication bias in meta-analysis. Biometrics 2000;56:455-63.

53. Gutierrez K. Group psychological intervention in assisted human reproduction. Rev Iberoam Fertil Reprod Hum 2008;25(Suppl 1):47-54.

54. Bjornsen K. Opening the door to fertility. retreat to fertile ground: sometimes the secret to getting pregnant lies in just getting away from it all. Altern Med 2006(88):80-1. http://search.ebscohost.com/ login.aspx?direct=true $\& \mathrm{db}=\mathrm{c} 8 \mathrm{~h} \& \mathrm{AN}=2009216983 \&$ site=ehost-live

55. Pengelly $P$, Inglis M, Cudmore L. Infertility: couples' experiences and the use of counselling in treatment centres. Psychodyn Couns 1995;1:507-24.

56. Stewart DE, Boydell KM, McCarthy K, et al. A prospective study of the effectiveness of brief professionally-led support groups for infertility patients. Int J Psychiatry Med 1992;22:173-82.

57. Landis JR, Koch GG. The measurement of observer agreement for categorical data. Biometrics 1977;33:159-74.

58. Nilforooshan P, Ahmadi A, Abedi MR, et al. Counseling based on interacting cognitive subsystems and its effect on anxiety of infertile couples. Pak J Psychol Res 2006;21:95-103. http://search. proquest.com.ez.statsbiblioteket.dk:2048/docview/621710509? accountid $=14468$

59. Schmidt L, Holstein BE, Christensen U, et al. Communication and coping as predictors of fertility problem stress: cohort study of 816 participants who did not achieve a delivery after 12 months of fertility treatment. Hum Reprod 2005;20:3248-56.

60. Galhardo A, Cunha M, Pinto-Gouveia J. Mindfulness-based program for infertility: Efficacy study. Fertil Steril 2013;100:1059-67.

61. Faramarzi M, Kheirkhah F, Esmaelzadeh S, et al. Is psychotherapy a reliable alternative to pharmacotherapy to promote the mental health of infertile women? A randomized clinical trial. Eur J Obstet Gynecol Reprod Biol 2008;141:49-53.

62. Lukse MP. The effect of group counseling on the frequency of grief reported by infertile couples. J Obstet Gynecol Neonatal Nurs 1985;14:67s-70s. http://search.ebscohost.com/login.aspx? direct $=$ true $\& d b=c 8 h \& A N=1986045607 \&$ site $=$ ehost-live,

63. Domar AD, Seibel MM, Benson $H$. The mind/body program for infertility: a new behavioral treatment approach for women with infertility. Fertil Steril 1990;53:246-9.

64. Domar AD, Zuttermeister PC, Seibel M, et al. Psychological improvement in infertile women after behavioral treatment-a replication. Fertil Steril 1992:58:144-7.

65. Emery M, Béran M-D, Darwiche J, et al. Results from a prospective, randomized, controlled study evaluating the acceptability and effects of routine pre-IVF counselling. Hum Reprod 2003;18:2647-53.

66. Lee S. Effects of using a nursing crisis intervention program on psychosocial responses and coping strategies of infertile women during in vitro fertilization. J Nurs Res 2003;11:197-207. http:// search.ebscohost.com/login.aspx? direct=true $\& \mathrm{db}=\mathrm{c} 8 \mathrm{~h} \& \mathrm{AN}=2004076404 \&$ site=ehost-live,

67. Chan $\mathrm{CHY}, \mathrm{Ng} \mathrm{EHY}$, Chan CLW, et al. Effectiveness of psychosocial group intervention for reducing anxiety in women undergoing in vitro fertilization: a randomized controlled study. Fertil Steril 2006;85:339-46.

68. Tuil WS, Verhaak CM, Braat DD, et al. Empowering patients undergoing in vitro fertilization by providing internet access to medical data. Fertil Steril 2007:88:361-8.

69. Lancastle D, Boivin J. A feasibility study of a brief coping intervention $(\mathrm{PRCl})$ for the waiting period before a pregnancy test during fertility treatment. Hum Reprod 2008;23:2299-307.

70. Mori A. Supporting stress management for women undergoing the early stages of fertility treatment: a cluster-randomized controlled trial. Jpn J Nurs Sci 2009;6:37-49.

71. Panagopoulou E, Montgomery A, Tarlatzis B. Experimental emotional disclosure in women undergoing infertility treatment: are drop outs better off? Soc Sci Med 2009;69:678-81.

72. Haemmerli K, Znoj H, Berger T. Internet-based support for infertile patients: a randomized controlled study. J Behav Med 2010;33:135-46

73. Hughes EG, Da Silva AM. A pilot study assessing art therapy as a mental health intervention for subfertile women. Hum Reprod 2011;26:611-15.

74. Matthiesen $\mathrm{S}$, Klonoff-Cohen $\mathrm{H}$, Zachariae $\mathrm{R}$, et al. The effect of an expressive writing intervention (EWI) on stress in infertile couples undergoing assisted reproductive technology (ART) treatment: a randomized controlled pilot study. Br J Health Psychol 2012;17:362-78

75. Mosalanejad L, Koolaee AK, Behbahani BM. Looking out for the secret wound: the effect of E-cognitive group therapy with emotional disclosure on the status of mental health in infertile women. Int J Fertil Steril 2012;6:87-94.

76. Mosalanejad L, Koolaee AK, Jamali S. Effect of cognitive behavioral therapy in mental health and hardiness of infertile women receiving assisted reproductive therapy (ART). Iran $J$ Reprod Med 2012;10:483-8.

77. Catoire P, Delaunay L, Dannappel T, et al. Hypnosis versus diazepam for embryo transfer: a randomized controlled study. Am J Clin Hypn 2013;55:378-86.

78. Domar AD, Clapp D, Slawsby E, et al. The impact of group psychological interventions on distress in infertile women. Health Psychol 2000;19:568-75. http://search.proquest.com.ez. statsbiblioteket.dk:2048/docview/619538260?accountid=14468,

79. O'Moore AM, O'Moore RR, Harrison RF. Psychosomatic aspects in idiopathic infertility: effects of treatment with autogenic training. J Psychosom Res 1983;27:145-51.

80. McQueeney D, Stanton A, Sigmon S. Efficacy of emotion-focused and problem-focused group therapies for women with fertility problems. J Behav Med 1997;20:313-31.

81. Koszycki D, Bisserbe J, Blier P, et al. Interpersonal psychotherapy versus brief supportive therapy for depressed infertile women: first pilot randomized controlled trial. Arch Womens Ment Health 2012;15:193-201.

82. Galletly C, Clark A, Tomlinson L, et al. Improved pregnancy rates for obese, infertile women following a group treatment program: an open pilot study. Gen Hosp Psychiatry 1996;18:192-5.

83. Cousineau TM, Green TC, Corsini E, et al Online psychoeducational support for infertile women: a randomized controlled trial. Hum Reprod 2008;23:554-66.

84. Noorbala AA, Ramazanzadeh F, Malekafzali $\mathrm{H}$, et al. Effects of a psychological intervention on depression in infertile couples. Int $J$ Gynecol Obstet 2008;101:248-52.

85. Sexton MB, Byrd MR, O'Donohue WT, et al. Web-based treatment for infertility-related psychological distress. Arch Womens Ment Health 2010;13:347-58.

86. Sarrel PM, DeCherney AH. Psychotherapeutic intervention for treatment of couples with secondary infertility. Fertil Steril 1985;43:897-900.

87. Hosaka T, Matsubayashi $\mathrm{H}$, Sugiyama $\mathrm{Y}$, et al. Effect of psychiatric group intervention on natural-killer cell activity and pregnancy rate. Gen Hosp Psychiatry 2002;24:353-6.

88. Levitas E, Parmet A, Lunenfeld E, et al. Impact of hypnosis during embryo transfer on the outcome of in vitro fertilization-embryo transfer: a case-control study. Fertil Steril 2006;85:1404-8.

89. Domar AD, Rooney KL, Wiegand B, et al. Impact of a group mind/ body intervention on pregnancy rates in IVF patients. Fertil Steril 2011;95:2269-73

90. Gorayeb R, Borsari AC, Rosa-e-Silva AC, et al. Brief cognitive behavioral intervention in groups in a Brazilian assisted reproduction program. Behav Med 2012;38:29-35.

91. Terzioglu F. Investigation into effectiveness of counseling on assisted reproductive techniques in turkey. J Psychosom Obstet Gynaecol 2001;22:133-41.

92. de Klerk C, Hunfeld JAM, Duivenvoorden HJ, et al. Effectiveness of a psychosocial counselling intervention for first-time IVF couples: a randomized controlled trial. Hum Reprod 2005;20:1333-8.

93. Vizheh M, Pakgohar M, Babaei G, et al. Effect of counseling on quality of marital relationship of infertile couples: a randomized, controlled trial (RCT) study. Arch Gynecol Obstet 2013; 287:583-9.

94. Chan $\mathrm{CH}$, Chan $\mathrm{CL}, \mathrm{Ng} \mathrm{EH}$, et al. Incorporating spirituality in psychosocial group intervention for women undergoing in vitro fertilization: a prospective randomized controlled study. Psychol Psychother 2012;85:356-73.

95. Domar A, Clapp D, Slawsby E, et al. Impact of group psychological interventions on pregnancy rates in infertile women. Fertil Steril 2000;73:805-11.

96. McNaughton-Cassill M, Bostwick JM, Arthur NJ, et al. Efficacy of brief couples support groups developed to manage the stress of in vitro fertilization treatment. Mayo Clin Proc 2002;77:1060-6. http:// search.ebscohost.com/login.aspx?direct=true \&db=c8h\&AN= 2003034100\&site=ehost-live

97. Galhardo A, Cunha M, Pinto-Gouveia J. Developing mindfulness and acceptance skills in infertility: the mindfulness based program for infertility. Aten Primaria 2013;45:143. 
98. Chen JH, Bierhals AJ, Prigerson HG, et al. Gender differences in the effects of bereavement-related psychological distress in health outcomes. Psychol Med 1999;29:367-80.

99. Navot D, Bergh RA, Williams MA, et al. Poor oocyte quality rather than implantation failure as a cause of age-related decline in female fertility. Lancet 1991;337:1375-7.

100. Dunson DB, Colombo B, Baird DD. Changes with age in the level and duration of fertility in the menstrual cycle. Hum Reprod 2002;17:1399-403.

101. Van den Broeck U, Emery M, Wischmann T, et al. Counselling in infertility: individual, couple and group interventions. Patient Educ Couns 2010;81:422-8.
102. Burns LH, Covington SN. Infertility counseling: a comprehensive handbook for clinicians. New York, NY, USA: Parthenon Publishing Group, 1999:xvii.

103. Boivin J, Appleton TC, Baetens P, et al. Guidelines for counselling in infertility: outline version. Hum Reprod 2001;16:1301-4.

104. Boivin J, Kentenich $\mathrm{H}$, eds. Guidelines for counselling in infertility. ESHRE Monographs: Oxford University Press, 2002.

105. Gameiro S, Boivin J, Domar A. Optimal in vitro fertilization in 2020 should reduce treatment burden and enhance care delivery for patients and staff. Fertil Steril 2013; 100:302-9. 